\title{
miRNA profiling of primary lung and head and neck squamous cell carcinomas: Addressing a diagnostic dilemma
}

\author{
Juan A. Muñoz-Largacha, MD, ${ }^{a}$ Adam C. Gower, $\mathrm{PhD},{ }^{\mathrm{b}}$ Praveen Sridhar, MD, ${ }^{\mathrm{c}}$ Anita Deshpande, MD, ${ }^{\mathrm{d}}$ \\ Carl J. O'Hara, MD, ${ }^{\mathrm{d}}$ Emiko Yamada, BA, ${ }^{\mathrm{e}}$ Tony E. Godfrey, PhD, ${ }^{\mathrm{e}}$ Hiran C. Fernando, MD, ${ }^{\mathrm{a}}$ and \\ Virginia R. Litle, $\mathrm{MD}^{\mathrm{a}}$
}

\begin{abstract}
Objective: To determine whether microRNA (miRNA) profiling of primary lung and head and neck squamous cell carcinomas could be useful to identify a specific miRNA signature that can be used to further discriminate between primary lung squamous carcinomas and metastatic lesions in patients with a history of head and neck squamous cell cancer.
\end{abstract}

Methods: Specimens of resected primary head and neck and lung squamous cell carcinomas were obtained from formalin-fixed, paraffin-embedded blocks. Paraffin blocks were sectioned and deparaffinized, and total RNA was isolated and profiled. Quantitative polymerase chain reaction was performed to verify array results.

Results: Twelve head and neck and 16 lung squamous cell carcinoma samples met quality control metrics and were included for analysis. Forty-eight miRNAs were differentially expressed $(P<.05)$ between the 2 groups. Of these, 30 were also significantly associated $(q<.25)$ with tumor type in 2 independent sets of primary head and neck and lung squamous carcinomas profiled by The Cancer Genome Atlas consortium, including miR-34a and miR-10a. The ratio of miR-10a and miR-10b was especially predictive of primary cancer site in all 3 data sets, with area under the (receiver operating characteristics) curve values ranging from 0.922 to 0.982 . Quantitative polymerase chain reaction confirmed the association of miR-34a expression and the miR-10:miR-10b ratio with tumor type.

Conclusions: MicroRNA expression may be useful for discriminating between head and neck and lung squamous cell carcinomas, including miR-34a and the miR-10a:miR-10b ratio. This differentiation has clinical importance because it could help determine the appropriate therapeutic approach. (J Thorac Cardiovasc Surg 2017;154:714-27)

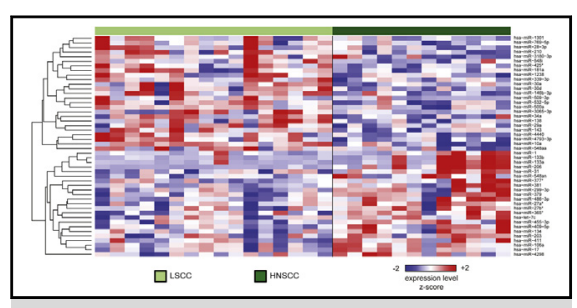

Expression of 48 miRNAs associated with squamous cancer type, including miR-34a and miR-10a.

\section{Central Message}

MicroRNA profiling is useful for discriminating between head and neck and lung squamous cell carcinomas, which could help in the selection of the appropriate therapeutic approach.

\section{Perspective}

The distinction between a primary lung squamous cell carcinoma and a metastatic lesion in a patient with a history of head and neck squamous cancer is challenging but essential to determine the proper treatment. MicroRNA profiling can help discriminate tumors from different tissues of origin. MiR-34a and miR$10 \mathrm{a}: 10 \mathrm{~b}$ may be especially useful in differentiating between a primary and a metastatic lesion.

See Editorial Commentary page 728.
From the ${ }^{\mathrm{a}}$ Division of Thoracic Surgery, Department of Surgery, ${ }^{\mathrm{b}}$ Clinical and Translational Science Institute, ${ }^{\mathrm{c}}$ General Surgery Training Program, ${ }^{\mathrm{d}}$ Department of Pathology and Laboratory Medicine, Boston Medical Center, and ${ }^{\mathrm{e}}$ Division of Surgical Research, Department of Surgery, Boston University School of Medicine, Boston, Mass.

This study was funded by the Department of Surgery of Boston University School of Medicine. A.C.G. was funded by Clinical and Translational Science Award UL1-TR001430.

Read at the 96th Annual Meeting of The American Association for Thoracic Surgery, Baltimore, Maryland, May 14-18, 2016.

Drs Muñoz-Largacha and Gower contributed equally to this article.

Received for publication May 24, 2016; revisions received Jan 31, 2017; accepted for publication Feb 25, 2017; available ahead of print May 8, 2017.

Address for reprints: Virginia R. Litle, MD, 88 East Newton St, Collamore Building Room C-7380, Boston, MA 02118 (E-mail: Virginia.litle@bmc.org).

0022-5223/ $\$ 36.00$

Copyright (c) 2017 by The American Association for Thoracic Surgery

http://dx.doi.org/10.1016/j.jtcvs.2017.02.071
Differentiating between a primary lung squamous cell carcinoma (LSCC) and a metastatic lesion in a patient with a history of head and neck squamous cell carcinoma (HNSCC) is challenging with the use of standard histopathology techniques. Both LSCC and HNSCC have several features in common, including similar histology,

Scanning this QR code will take you to supplemental tables for this article. 


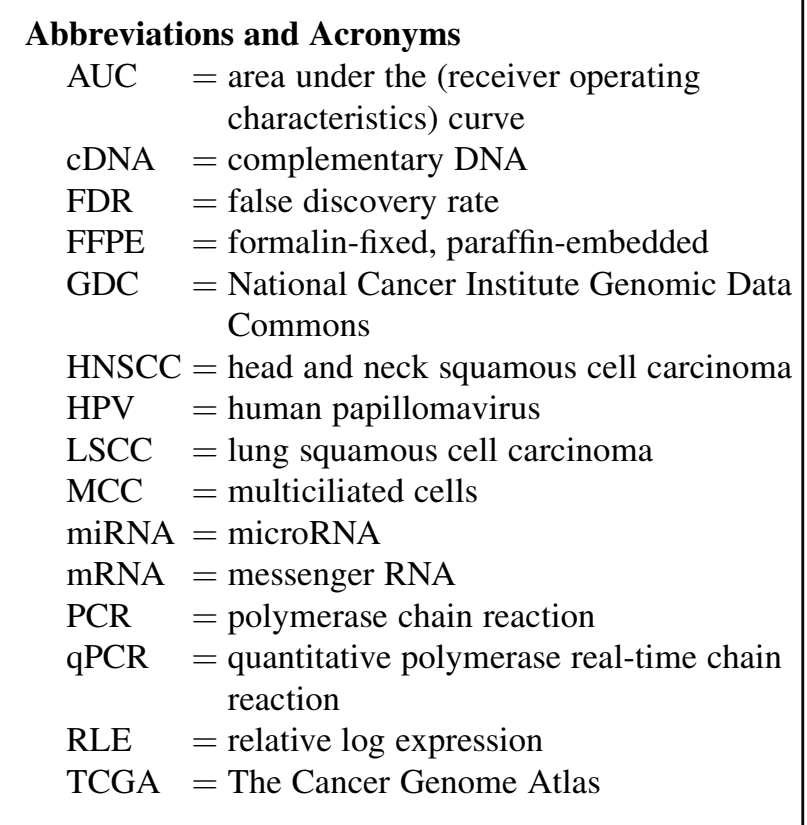

epithelial cells of origin, and association with tobacco exposure. $^{1,2}$ Even though the rate of metastasis from HNSCC is relatively low and may depend on locoregional control and lymph node status, the lungs frequently are involved, accounting for up to $70 \%$ to $85 \%$ of HNSCC metastatic cases. ${ }^{3}$ Patients with a history of HNSCC who present with new lung lesions can therefore represent a challenge for surgeons who must plan the appropriate therapeutic approach. In patients with locoregional control of their HNSCC with metastasis to the lung, a complete, nonanatomical or wedge resection is indicated. However, the standard of care for primary LSCC is an anatomical resection, which includes a segmentectomy, lobectomy, or pneumonectomy, which have greater morbidity and perioperative mortality compared with nonanatomic resections. 4

MicroRNAs (miRNAs) regulate gene expression and are involved in multiple biologic processes, including cell differentiation, proliferation, apoptosis, and metastasis. ${ }^{5}$ Their role in the development and pathogenesis of cancer also has been described, ${ }^{6}$ and it is known that miRNAs can act as tumor suppressors and oncogenes. ${ }^{5}$ It also has been shown that miRNAs can be expressed differentially between different tissues and tumor types, and their expression can differentiate tumors with different developmental origin. $^{7}$ MiRNA profiling is a well-accepted method for the identification of specific miRNAs related to different diseases and types of cancer. ${ }^{5,8}$ This could be useful in the diagnosis of metastatic cancer of unknown primary site $^{8}$ and in tumors with similar histopathologic findings in which a diagnosis under light microscopy can be challenging.
An accurate diagnosis of a new lung lesion in a patient with history of HNSCC is essential because this will help determine the appropriate therapeutic approach and surgical resection extent. In this study, we aimed to determine whether miRNA profiling is useful to differentiate between primary LSCC and HNSCC to identify characteristic features that are useful for differentiating between a primary LSCC and a metastatic lesion in a patient with history of HNSCC.

\section{MATERIALS AND METHODS \\ Sample Identification, Formalin-Fixed, Paraffin- Embedded (FFPE) Processing, and RNA Isolation}

Institutional review board approval was granted for this study (IRB ID \#H-34408). LSCC and HNSCC specimens were obtained from FFPE blocks of surgically resected tissue from patients at Boston Medical Center. Of the 89 LSCC cases identified between September 2002 and April 2013 and the 44 HNSCC cases identified between March 2003 and May 2013, 18 LSCC and 17 HNSCC specimens from patients without a previous history of cancer or chemo/radiotherapy were selected for analysis. LSCC tissue was resected via segmentectomy, lobectomy, and pneumonectomy. Two patients with poor pulmonary function were not good candidates for anatomical resections and underwent wedge resection of their lung nodules. HNSCC were taken from the larynx, oropharynx, oral cavity, and floor of the mouth. Human papillomavirus (HPV) status was assessed by P16 staining in all HNSCC cases. The FFPE samples were sectioned with a microtome into four $10-\mu \mathrm{m}$ sections and placed in a capped $1.5-\mathrm{mL}$ centrifuge tube. Before sectioning, each specimen was examined with hematoxylin and eosin staining by a pathologist to confirm tumor histopathology and to ensure that the specimen contained at least $70 \%$ tumor. Sectioned FFPE samples were deparaffinized with QIAGEN Deparaffinization Solution (QIAGEN, Germantown, Md), and total RNA was isolated with the QIAGEN miRNeasy FFPE kit and protocol. RNA samples were quantified with a NanoDrop spectrophotometer (NanoDrop Products, Wilmington, Del), and RNA integrity examined with the Agilent Bioanalyzer (Agilent, Santa Clara, Calif).

\section{Normalization and Quality Assessment}

Total RNA was profiled with the Affymetrix GeneChip miRNA 3.0 array (Thermo Fisher Scientific, Waltham, Mass). Raw CEL files were normalized to produce probeset-level expression values and detection (Present/Absent) calls for all 5639 human and control probesets (including 1733 mature human miRNAs) with Affymetrix Expression Console (version 1.3.0.187), with the Robust Multiarray Average and Detection Above BackGround. Expression Console also was used to generate relative log expression (RLE) values as a measure of sample quality: a distribution of RLE values skewed upwards often indicates that the expression values were inflated by the normalization process. Samples with a mean RLE $\geq 0.5$ or in which $\leq 20 \%$ of all miRNAs were called Present were excluded from analysis. Any miRNAs with spurious expression, ie, those that were not called Present in at least $25 \%$ of all samples, also were excluded from analysis.

\section{The Cancer Genome Atlas (TCGA) Data}

Preprocessed miRNA profiling data for primary tumors from 478 LSCC and 523 HNSCC TCGA samples, profiled by using Illumina Genome Analyzer (Illumina Inc, San Diego, Calif) or HiSeq instruments, were obtained from the National Cancer Institute's Genomic Data Commons (GDC) Portal at https://gdc-portal.nci.nih.gov. The data matrices used comprised counts that had been generated for 1881 human miRNAs in each sample via the use of miRBase version 21 and human genome build 
hg38. Biospecimen and clinical annotation also were obtained from the GDC Portal as XML files associated with each case (patient). The HPV status of each sample was obtained from the "hpv_status" field in the biospecimen annotation, and diagnoses, histology, staging information, and clinical history were obtained from the clinical annotation. Additional biospecimen and clinical notes not present in these XML files were obtained from the GDC Portal in a "metadata" JSON file that accompanied the download. Two LSCC cases (TCGA-90-6837 and TCGA-56-8623) were excluded from analysis because they were annotated as having a possible tumor/normal swap or other sample-related issues. Patients were excluded from analysis where indicated based on the binary "history_of neoadjuvant_treatment" and "other_dx" clinical parameters, or if any clinical notes in the metadata indicated a positive or unknown history of malignancy and/or chemotherapy. Clinical history of radiation therapy was not annotated for the majority of cases, and so this parameter was not considered as an exclusion criterion. Counts were normalized to the total number of counts in each sample to obtain reads per million reads mapped values. Reads per million reads mapped values were transformed before statistical analysis by adding a pseudocount of 1 and $\log 2$-transforming the result to render them more likely to meet the assumptions of Student $t$ test.

\section{Statistical Analysis}

Differential expression was assessed by performing a Student $t$ test on the coefficients of simple linear models created with the "limma" $\mathrm{R}$ package (version 3.14.4). Correction for multiple hypothesis testing was performed with the Benjamini-Hochberg false discovery rate (FDR). Hierarchical clustering was performed with complete linkage and Euclidean distance metric, using expression values $\mathrm{z}$-normalized to a mean of zero and standard deviation of one across all samples. All microarray analyses were performed using the $\mathrm{R}$ environment for statistical computing (version 2.15.1; the R Foundation for Statistical Computing, Vienna, Austria).

\section{Quantitative Real-Time Polymerase Chain Reaction (qPCR)}

Complementary DNA (cDNA) was made with the TaqMan Advanced miRNA cDNA Synthesis kit and protocol. Quantitative real-time qPCR (qPCR) was performed with TaqMan Advanced miRNA Assays (Thermo Fisher Scientific) and Fast Advanced Master Mix (Thermo Fisher Scientific) using the StepOnePlus Real-Time PCR System (Applied Biosystems, Foster City, Calif). Three replicates of cDNA were made for each sample and duplicate qPCRs were set up for each cDNA. Within each PCR plate, pooled RNA from 8 samples (4 LSCC, 4 HNSCC) was included as a reference (calibrator), along with negative controls in which reverse transcriptase or template were excluded. TaqMan Advanced miRNA Assays were used to measure hsa-miR-191-5p (\#477952_mir) and hsa-miR-361-5p (\#478056_mir) as endogenous controls based on the array data. Relative expression of miR-34a was determined as normalized relative quantity using the method of Hellemans and colleagues, ${ }^{9}$ that is, fold expression of miR-34a relative to the reference sample divided by the geometric mean of the fold expression of each endogenous control relative to the reference sample. The miR-10a:miR$10 \mathrm{~b}$ ratio was computed by raising 2 to the power of the difference between the $\Delta \mathrm{Ct}$ values for miR-10a and miR-10b (computed relative to the reference sample).

Spike-in controls were performed to establish primer specificity between miR-10a and miR-10b because of their sequence similarity. Yeast total RNA (Ambion; Thermo Fisher Scientific) was used as background and miR-10a and miR-10b RNA oligonucleotides (Integrated DNA Technologies, Coralville, Iowa) were used for the spike-in. Three replicates of cDNA were done for each sample, and 3 replicates of each cDNA were used for the PCR. Reactions in which reverse transcriptase or spike-in RNAs were excluded were used as negative controls.

\section{RESULTS}

Demographic and histopathologic information pertaining to the samples from our cohort and from primary tumors profiled by TCGA are summarized in Table 1. In our cohort, the median time from resection to RNA isolation did not differ across tumor types: 29 months (interquartile range 10.8-39 months) for the LSCC samples, and 20 months (interquartile range 14.2-27.8 months) for the HNSCC samples $(P=.46$, Student $t$ test).

A total of 48 miRNAs were detected in at least $25 \%$ of all samples (see Materials and Methods) and had nominally significant differential expression between HNSCC and LSCC $(P<.05)$, with 4 (miR-206, miR-10a, miR-4440, and miR-4793) passing multiple hypothesis correction (FDR $q<0.25$ ) (Figure 1, Table 2, and Online Data Supplement 1). Of the 25 miRNAs with significantly greater expression in LSCC, the difference was the most significant in the highly conserved miR-10a $\left(P=4.5 \times 10^{-5}\right.$, FDR $q=0.039$ ), and intriguingly, the expression of the closely related miRNA miR-10b (which differs from miR-10a by only one nucleotide but shares its seed sequence) trended strongly in the opposite direction (1.7-fold greater in HNSCC, $P=.08$ ). Another highly conserved miRNA, miR-34a, also was expressed at significantly greater levels in LSCC $(P=.004)$. Of the 48 miRNAs, 30 also were significantly (FDR $q<0.25$ ) associated with tumor type in the same direction in both TCGA datasets (Table 2 and Online Data Supplements 2 and 3). Intriguingly, miR-10a was the most significantly differentially expressed miRNA whose expression was greater in LSCC in both TCGA datasets, indicating that this locus is very robustly associated with tumor type, and the expression of miR-10b also was associated strongly with tumor type in both TCGA datasets. Finally, miR-34a also was concordant in the TCGA datasets, ranking among the top $12 \%$ most significantly upregulated miRNAs in LSCC.

To assess possible sources of heterogeneity within HNSCC tumors, the TCGA datasets were used to perform a one-way analysis of variance with respect to anatomic site and to perform Student $t$ tests between stage IV and non-stage-IV disease (Online Data Supplements 2 and $3)$. The expression of miR-10a was associated strongly with anatomic site (FDR $q<0.25$ ) in both datasets, whereas this held true for miR-34a in only the HiSeq dataset. The expression of miR-10b was not associated with site in either dataset (FDR $q>0.25$ ). None of these 3 miRNAs was associated with stage IV disease in HNSCC in either dataset (all FDR $q>0.25$ ).

\section{Predictive Power of miR-10a:miR-10b Ratio}

Given that the 2 highly related miRNAs miR-10a and miR-10b were strongly differentially expressed in opposing directions between LSCC and HNSCC in all 3 datasets, the 
TABLE 1. Characteristics of the patient samples analyzed in this study

\begin{tabular}{|c|c|c|c|c|c|c|c|}
\hline \multicolumn{4}{|c|}{ LSCC } & \multicolumn{4}{|c|}{ HNSCC } \\
\hline & $\begin{array}{c}\text { Affymetrix } \\
\text { miRNA v3.0 } \\
\end{array}$ & $\begin{array}{c}\text { Illumina } \\
\text { GA }\end{array}$ & $\begin{array}{c}\text { Illumina } \\
\text { HiSeq }\end{array}$ & & $\begin{array}{c}\text { Affymetrix } \\
\text { miRNA v3.0 }\end{array}$ & $\begin{array}{c}\text { Illumina } \\
\text { GA }\end{array}$ & $\begin{array}{c}\text { Illumina } \\
\text { HiSeq }\end{array}$ \\
\hline $\mathrm{n}$ & 16 & 136 & 340 & $\mathrm{n}$ & 12 & 37 & 486 \\
\hline Sex, $\%$ male & 75 & 71.3 & 75.0 & Sex, $\%$ male & 50.0 & 75.7 & 72.8 \\
\hline Age, mean $\pm \mathrm{SD}$ & $69.5 \pm 8.6$ & $67.3 \pm 8.6$ & $67.5 \pm 8.7$ & Age, mean $\pm S D$ & $64.9 \pm 16.3$ & $60.1 \pm 13.9$ & $61.0 \pm 11.8$ \\
\hline \multicolumn{8}{|l|}{ Smoking history } \\
\hline Mean pack-years & 74.1 & 56.9 & 51.9 & Mean pack-years & 36.6 & 44.5 & 45.8 \\
\hline Current smoker & 3 & 24 & 105 & Current smoker & 1 & 19 & 157 \\
\hline Former smoker & 13 & 108 & 207 & Former smoker & 5 & 13 & 198 \\
\hline Never smoker & 0 & 4 & 12 & Never smoker & 6 & 5 & 116 \\
\hline Unknown & 0 & 0 & 16 & Unknown & 0 & 0 & 15 \\
\hline \multicolumn{8}{|l|}{ Location of tumor } \\
\hline Bronchial & 1 & 2 & 8 & Alveolar ridge & 0 & 1 & 17 \\
\hline LLL & 1 & 28 & 42 & Base of tongue & 0 & 3 & 24 \\
\hline LUL & 6 & 37 & 91 & Buccal mucosa & 1 & 3 & 19 \\
\hline LUL and LLL & 2 & 0 & 0 & Floor of mouth & 1 & 4 & 58 \\
\hline RLL & 1 & 29 & 77 & Hard palate & 0 & 1 & 6 \\
\hline RML & 0 & 4 & 11 & Hypopharynx & 0 & 1 & 9 \\
\hline RUL & 5 & 34 & 95 & Larynx & 6 & 11 & 106 \\
\hline Other & 0 & 0 & 11 & Lip & 0 & 0 & 3 \\
\hline \multirow[t]{5}{*}{ Unknown } & 0 & 2 & 5 & Mandible & 1 & 0 & 0 \\
\hline & & & & Oral cavity & 1 & 3 & 70 \\
\hline & & & & Oral tongue & 0 & 6 & 125 \\
\hline & & & & Oropharynx & 2 & 0 & 9 \\
\hline & & & & Tonsil & 0 & 4 & 40 \\
\hline \multicolumn{8}{|c|}{ Surgical procedure/type of resection } \\
\hline Wedge & 2 & & & Total laryngectomy & 5 & & \\
\hline Segmentectomy & 1 & & & Supraglottic laryngectomy & 1 & & \\
\hline Lobectomy & 8 & & & Oropharyngeal resection & 3 & & \\
\hline \multirow[t]{2}{*}{ Pneumonectomy } & 5 & & & Mandibulectomy & 2 & & \\
\hline & & & & Excisional biopsy buccal mucosa & 1 & & \\
\hline \multicolumn{8}{|l|}{ HPV status } \\
\hline & & & & Positive & 4 & 10 & 87 \\
\hline & & & & Negative & 4 & 23 & 397 \\
\hline & & & & Indeterminate & 4 & 0 & 2 \\
\hline \multicolumn{8}{|c|}{ Anatomic stage (TNM classification) } \\
\hline Stage I & 0 & 0 & 3 & Stage I & 1 & 1 & 26 \\
\hline Stage IA & 6 & 19 & 64 & Stage II & 1 & 2 & 71 \\
\hline Stage IB & 4 & 53 & 90 & Stage III & 0 & 3 & 76 \\
\hline Stage II & 0 & 0 & 3 & Stage IVA & 8 & 24 & 233 \\
\hline Stage IIA & 2 & 6 & 56 & Stage IVB & 2 & 2 & 10 \\
\hline Stage IIB & 1 & 25 & 67 & Stage IVC & 0 & 0 & 1 \\
\hline Stage III & 0 & 0 & 3 & Unknown & 0 & 5 & 69 \\
\hline Stage IIIA & 3 & 19 & 42 & & & & \\
\hline Stage IIIB & 0 & 10 & 6 & & & & \\
\hline Stage IV & 0 & 3 & 3 & & & & \\
\hline Unknown & 0 & 1 & 3 & & & & \\
\hline \multicolumn{8}{|l|}{ Differentiation grade } \\
\hline Well & 1 & & & Well & 3 & & \\
\hline Moderate & 15 & & & Moderate & 5 & & \\
\hline Moderate to poor & 0 & & & Moderate to poor & 1 & & \\
\hline Poor & 0 & & & Poor & 3 & & \\
\hline
\end{tabular}


TABLE 1. Continued

\begin{tabular}{|c|c|c|c|c|c|c|c|}
\hline \multicolumn{4}{|c|}{ LSCC } & \multicolumn{4}{|c|}{ HNSCC } \\
\hline & $\begin{array}{c}\text { Affymetrix } \\
\text { miRNA v3.0 }\end{array}$ & $\begin{array}{c}\text { Illumina } \\
\text { GA }\end{array}$ & $\begin{array}{c}\text { Illumina } \\
\text { HiSeq }\end{array}$ & & $\begin{array}{c}\text { Affymetrix } \\
\text { miRNA v3.0 }\end{array}$ & $\begin{array}{c}\text { Illumina } \\
\text { GA }\end{array}$ & $\begin{array}{c}\text { Illumina } \\
\text { HiSeq }\end{array}$ \\
\hline \multicolumn{8}{|c|}{ Time from resection to RNA isolation } \\
\hline Months, median (IQR) & $29(10.8-39)$ & & & Months, median (IQR) & $20(14.2-27.8)$ & & \\
\hline \multicolumn{8}{|c|}{ Meets exclusion criteria (previous, synchronous, or unknown history of malignancy or neoadjuvant treatment) } \\
\hline Yes & 0 & 24 & 49 & Yes & 0 & 2 & 37 \\
\hline
\end{tabular}

Demographic and histopathologic parameters are tabulated within each platform and tumor type. $L S C C$, Lung squamous cell carcinoma; HNSCC, head and neck squamous cell carcinoma; miRNA, microRNA; GA, Genome Analyzer; $L L L$, left lower lobe; $L U L$, left upper lobe; $R L L$, right lower lobe; $R M L$, right middle lobe; $R U L$, right upper lobe; $H P V$, human papillomavirus; $I Q R$, interquartile range.

power of the miR-10a:miR-10b ratio as a discriminator between the two tumor types was examined (Table 3). The performance of this ratio was examined with respect to (1) the platform used, (2) exclusion criteria (previous or unknown history of malignancy, chemotherapy or [in our cohort] radiation therapy; see Materials and Methods), (3) HPV status of HNSCC tumors, and (4) inclusion or exclusion of non-stage-IV HNSCC. The miR-10a:miR$10 \mathrm{~b}$ ratio had substantially greater predictive power in the samples that were profiled with the HiSeq platform (area under the [receiver operating characteristics] curve [AUC] $\sim 0.98$, accuracy $\sim 92 \%$ ) than in those profiled with the Genome Analyzer platform (AUC 0.97, accuracy $\sim 82 \%$ ) or by microarray (AUC $<0.97$, accuracy $79 \%$ $85 \%$ ). Application of the exclusion criteria or removal of non-stage-IV HNSCC had only minor effects on the predictive performance of the ratio in the TCGA cohorts.
However, consideration of HPV status produced mixed results, leading to little impact in the TCGA datasets, but a substantial improvement in the AUC in the microarray dataset (from 0.92 to 0.97 ).

\section{Quantitative Real-Time PCR}

We then used qPCR to measure the expression of miR-34a, miR-10a, and miR-10b in our set of 28 samples. The normalized expression of miR-34a agreed well with the relative expression of miR-34a (normalized to the same controls) as measured by microarray (Figure 2; Pearson $r=0.65$ ), and, accordingly, the expression was significantly different between LSCC and HNSCC (Student $P=.049)$. The miR-10a:miR-10b ratio also was in good agreement with that determined by microarray (Figure 3; Pearson $r=0.81$ ). Interestingly, however, the magnitude and direction of the miR-10a:miR-10b ratio were rather

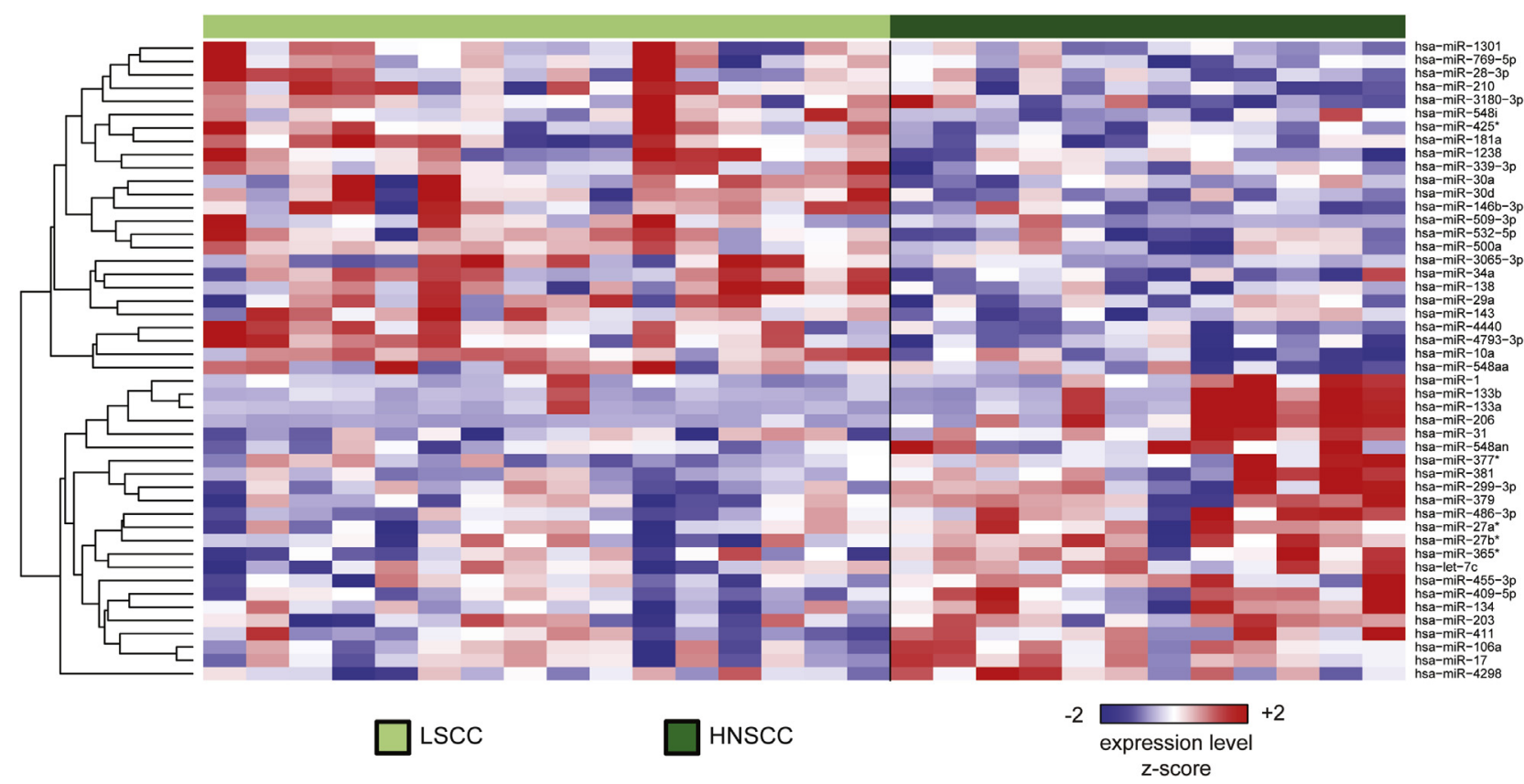

FIGURE 1. Heatmap of miRNAs differentially expressed between LSCC and HNSCC. Red and blue indicate expression values $\geq 2$ standard deviations above and below, respectively, the row-wise mean (white) computed across all samples. LSCC and HNSCC samples are indicated in light and dark green, respectively. $L S C C$, Lung squamous cell carcinoma; HNSCC, head and neck squamous cell carcinoma. 
TABLE 2. MicroRNAs with differential expression between LSCC and HNSCC

\begin{tabular}{|c|c|c|c|c|c|c|c|c|c|c|c|c|c|}
\hline \multicolumn{7}{|c|}{ Affymetrix miRNA v3.0 } & \multirow[b]{2}{*}{$\begin{array}{c}\text { miRBase } \\
\text { v21 } \\
\text { transcript } \\
\text { ID } \\
\end{array}$} & \multicolumn{3}{|c|}{ Illumina GA } & \multicolumn{3}{|c|}{ Illumina HiSeq } \\
\hline $\begin{array}{c}\text { Probeset } \\
\text { ID } \\
\end{array}$ & $\begin{array}{c}\text { Transcript } \\
\text { ID(s) } \\
\end{array}$ & $\begin{array}{c}\text { Alignment(s) } \\
\text { (hg19) }\end{array}$ & $\begin{array}{c}\text { Probeset } \\
\text { Present } \\
(\% \\
\text { samples }) \\
\end{array}$ & $\begin{array}{c}\text { Fold } \\
\text { Change } \\
\end{array}$ & $\begin{array}{c}P \\
\text { value } \\
\end{array}$ & $\begin{array}{c}\text { FDR } \\
q \\
\end{array}$ & & $\begin{array}{c}\text { Fold } \\
\text { Change } \\
\end{array}$ & $P$ value & FDR $q$ & $\begin{array}{c}\text { Fold } \\
\text { Change } \\
\end{array}$ & $P$ value & FDR $q$ \\
\hline $\begin{array}{l}\text { hsa-miR- } \\
\text { 206_st }\end{array}$ & $\begin{array}{l}\text { hsa-mir- } \\
206\end{array}$ & $\begin{array}{c}6: 52009147- \\
52009232 \\
(+)\end{array}$ & 36 & 448.9 & .000017 & 0.030 & $\begin{array}{l}\text { hsa-mir- } \\
206\end{array}$ & 878.0 & $\begin{array}{l}1.93 \times \\
10^{-44}\end{array}$ & $\begin{array}{r}2.83 \times \\
10^{-41}\end{array}$ & 782.5 & $\begin{array}{l}1.76 \times \\
10^{-125}\end{array}$ & $\begin{array}{r}1.45 \times \\
10^{-122}\end{array}$ \\
\hline $\begin{array}{l}\text { hsa-miR- } \\
\quad 486-3 p_{-} \\
\text {st }\end{array}$ & $\begin{array}{c}\text { hsa-mir- } \\
486\end{array}$ & $\begin{array}{l}8: 41517959- \\
41518026 \\
(-)\end{array}$ & 64 & 4.4 & .0022 & 0.68 & $\begin{array}{r}\text { hsa-mir- } \\
486-1\end{array}$ & 2.2 & $\begin{array}{r}2.78 \times \\
10^{-7}\end{array}$ & $\begin{array}{r}1.20 \times \\
10^{-5}\end{array}$ & 1.4 & $\begin{array}{r}9.69 \times \\
10^{-9}\end{array}$ & $\begin{array}{r}5.61 \times \\
10^{-8}\end{array}$ \\
\hline \multirow[t]{2}{*}{$\begin{array}{l}\text { hsa-miR- } \\
\text { 133a_st }\end{array}$} & $\begin{array}{l}\text { hsa-mir- } \\
\text { 133a-1 }\end{array}$ & $\begin{array}{c}\text { 18:19405659- } \\
19405746 \\
(-)\end{array}$ & 29 & 16.6 & .0058 & 0.69 & $\begin{array}{l}\text { hsa-mir- } \\
\text { 133a-1 }\end{array}$ & 103.4 & $\begin{array}{c}4.87 \times \\
10^{-21}\end{array}$ & $\begin{array}{c}1.79 \times \\
10^{-18}\end{array}$ & 56.2 & $\begin{array}{c}1.89 \times \\
10^{-47}\end{array}$ & $\begin{array}{l}2.08 \times \\
10^{-45}\end{array}$ \\
\hline & $\begin{array}{l}\text { hsa-mir- } \\
133 a-2\end{array}$ & $\begin{array}{l}\text { 20:61162119- } \\
\quad 61162220 \\
(+)\end{array}$ & & & & & $\begin{array}{l}\text { hsa-mir- } \\
133 a-2\end{array}$ & 103.8 & $\begin{array}{l}1.35 \times \\
10^{-18}\end{array}$ & $\begin{array}{r}3.96 \times \\
10^{-16}\end{array}$ & 56.3 & $\begin{array}{r}3.52 \times \\
10^{-45}\end{array}$ & $\begin{array}{r}3.41 \times \\
10^{-43}\end{array}$ \\
\hline $\begin{array}{c}\text { hsa-miR- } \\
\text { 31_st }\end{array}$ & $\begin{array}{l}\text { hsa-mir- } \\
31\end{array}$ & $\begin{array}{l}9: 21512114- \\
21512184 \\
(-)\end{array}$ & 100 & 4.4 & .0067 & 0.69 & $\begin{array}{l}\text { hsa-mir- } \\
31\end{array}$ & 2.2 & .000024 & 0.00054 & 3.6 & $\begin{array}{r}6.77 \times \\
10^{-39}\end{array}$ & $\begin{array}{r}3.19 \times \\
10^{-37}\end{array}$ \\
\hline $\begin{array}{l}\text { hsa-miR- } \\
\text { 365-star_ } \\
\text { st }\end{array}$ & $\begin{array}{r}\text { hsa-mir- } \\
365-2\end{array}$ & $\begin{array}{c}\text { 17:29902430- } \\
29902540 \\
(+)\end{array}$ & 75 & 1.6 & .0071 & 0.69 & $\begin{array}{l}\text { hsa-mir- } \\
\text { 365b }\end{array}$ & 1.6 & .000011 & 0.00028 & 1.3 & $\begin{array}{r}7.03 \times \\
10^{-9}\end{array}$ & $\begin{array}{r}4.13 \times \\
10^{-8}\end{array}$ \\
\hline $\begin{array}{l}\text { hsa-miR- } \\
\quad 409-5 p_{-} \\
\text {st }\end{array}$ & $\begin{array}{c}\text { hsa-mir- } \\
409\end{array}$ & $\begin{array}{c}14: 101531637- \\
101531715 \\
(+)\end{array}$ & 64 & 2.4 & .0074 & 0.69 & $\begin{array}{c}\text { hsa-mir- } \\
409\end{array}$ & -1.2 & .13 & 0.37 & -1.3 & .79 & 0.85 \\
\hline $\begin{array}{l}\text { hsa-miR- } \\
\text { 133b_st }\end{array}$ & $\begin{array}{c}\text { hsa-mir- } \\
\text { 133b }\end{array}$ & $\begin{array}{l}6: 52013721- \\
52013839 \\
(+)\end{array}$ & 32 & 17.8 & .010 & 0.69 & $\begin{array}{c}\text { hsa-mir- } \\
\text { 133b }\end{array}$ & 78.0 & $\begin{array}{c}1.93 \times \\
10^{-21}\end{array}$ & $\begin{array}{c}9.44 \times \\
10^{-19}\end{array}$ & 51.9 & $\begin{array}{l}8.57 \times \\
10^{-49}\end{array}$ & $\begin{array}{r}1.18 \times \\
10^{-46}\end{array}$ \\
\hline $\begin{array}{l}\text { hsa-miR- } \\
\text { 299-3p_ } \\
\text { st }\end{array}$ & $\begin{array}{l}\text { hsa-mir- } \\
299\end{array}$ & $\begin{array}{c}14: 101490131- \\
101490193 \\
(+)\end{array}$ & 75 & 2.2 & .015 & 0.75 & $\begin{array}{c}\text { hsa-mir- } \\
299\end{array}$ & 1.5 & .000078 & 0.0014 & 1.3 & $\begin{array}{l}6.11 \times \\
10^{-15}\end{array}$ & $\begin{array}{r}6.68 \times \\
10^{-14}\end{array}$ \\
\hline $\begin{array}{l}\text { hsa-miR- } \\
\quad 455-3 p_{-} \\
\text {st }\end{array}$ & $\begin{array}{l}\text { hsa-mir- } \\
455\end{array}$ & $\begin{array}{l}9: 116971714- \\
116971809 \\
(+)\end{array}$ & 100 & 1.8 & .017 & 0.76 & $\begin{array}{l}\text { hsa-mir- } \\
455\end{array}$ & 2.0 & $\begin{array}{c}6.69 \times \\
10^{-11}\end{array}$ & $\begin{array}{r}5.78 \times \\
10^{-9}\end{array}$ & 1.2 & .00082 & 0.0024 \\
\hline $\begin{array}{l}\text { hsa-miR- } \\
\text { 381_st }\end{array}$ & $\begin{array}{c}\text { hsa-mir- } \\
381\end{array}$ & $\begin{array}{l}14: 101512257- \\
101512331 \\
(+)\end{array}$ & 54 & 4.0 & .017 & 0.76 & $\begin{array}{c}\text { hsa-mir- } \\
381\end{array}$ & 1.0 & .016 & 0.10 & 1.2 & .00009 & 0.00031 \\
\hline $\begin{array}{r}\text { hsa-miR- } \\
411 \_s t\end{array}$ & $\begin{array}{c}\text { hsa-mir- } \\
411\end{array}$ & $\begin{array}{c}14: 101489662- \\
101489757 \\
(+)\end{array}$ & 36 & 1.7 & .020 & 0.78 & $\begin{array}{c}\text { hsa-mir- } \\
411\end{array}$ & 1.2 & .0024 & 0.022 & 1.2 & $\begin{array}{r}2.22 \times \\
10^{-6}\end{array}$ & $\begin{array}{r}9.87 \times \\
10^{-6}\end{array}$ \\
\hline $\begin{array}{l}\text { hsa-miR- } \\
\text { 548an_st }\end{array}$ & $\begin{array}{r}\text { hsa-mir- } \\
548 \mathrm{an}\end{array}$ & $\begin{array}{c}\mathrm{X}: 105883044- \\
105883126 \\
(+)\end{array}$ & 25 & 1.4 & .021 & 0.78 & $\begin{array}{r}\text { hsa-mir- } \\
548 \mathrm{an}\end{array}$ & 1.0 & .11 & 0.33 & -1.0 & .81 & 0.87 \\
\hline $\begin{array}{r}\text { hsa-miR- } \\
\text { 379_st }\end{array}$ & $\begin{array}{c}\text { hsa-mir- } \\
379\end{array}$ & $\begin{array}{c}14: 101488403- \\
101488469 \\
(+)\end{array}$ & 89 & 2.7 & .021 & 0.78 & $\begin{array}{c}\text { hsa-mir- } \\
379\end{array}$ & -1.1 & .13 & 0.37 & 1.2 & $\begin{array}{r}5.25 \times \\
10^{-8}\end{array}$ & $\begin{array}{r}2.80 \times \\
10^{-7}\end{array}$ \\
\hline $\begin{array}{r}\text { hsa-miR- } \\
\text { 134_st }\end{array}$ & $\begin{array}{c}\text { hsa-mir- } \\
134\end{array}$ & $\begin{array}{c}14: 101521024- \\
101521096 \\
(+)\end{array}$ & 96 & 2.0 & .025 & 0.78 & $\begin{array}{c}\text { hsa-mir- } \\
134\end{array}$ & -1.2 & .042 & 0.19 & -1.1 & .021 & 0.047 \\
\hline $\begin{array}{r}\text { hsa-miR- } \\
\text { 203_st }\end{array}$ & $\begin{array}{c}\text { hsa-mir- } \\
203\end{array}$ & $\begin{array}{c}14: 104583742- \\
104583851 \\
(+)\end{array}$ & 100 & 2.0 & .028 & 0.80 & $\begin{array}{c}\text { hsa-mir- } \\
203 a\end{array}$ & 3.4 & $\begin{array}{c}8.22 \times \\
10^{-11}\end{array}$ & $\begin{array}{r}6.71 \times \\
10^{-9}\end{array}$ & 3.0 & $\begin{array}{c}8.52 \times \\
10^{-39}\end{array}$ & $\begin{array}{c}3.90 \times \\
10^{-37}\end{array}$ \\
\hline $\begin{array}{l}\text { hsa-miR- } \\
\text { 27b-star } \\
\text { st } \\
\end{array}$ & $\begin{array}{c}\text { hsa-mir- } \\
27 \mathrm{~b}\end{array}$ & $\begin{array}{l}9: 97847727- \\
97847823 \\
(+)\end{array}$ & 100 & 1.8 & .035 & 0.83 & $\begin{array}{l}\text { hsa-mir- } \\
27 \mathrm{~b}\end{array}$ & 1.4 & .07 & 0.26 & 1.2 & .0034 & 0.0091 \\
\hline
\end{tabular}


TABLE 2. Continued

\begin{tabular}{|c|c|c|c|c|c|c|c|c|c|c|c|c|c|}
\hline \multicolumn{7}{|c|}{ Affymetrix miRNA v3.0 } & \multirow[b]{2}{*}{$\begin{array}{c}\text { miRBase } \\
\text { v21 } \\
\text { transcript } \\
\text { ID } \\
\end{array}$} & \multicolumn{3}{|c|}{ Illumina GA } & \multicolumn{3}{|c|}{ Illumina HiSeq } \\
\hline $\begin{array}{c}\text { Probeset } \\
\text { ID }\end{array}$ & $\begin{array}{c}\text { Transcript } \\
\text { ID(s) }\end{array}$ & $\begin{array}{l}\text { Alignment(s) } \\
\text { (hg19) }\end{array}$ & $\begin{array}{c}\text { Probeset } \\
\text { Present } \\
(\% \\
\text { samples })\end{array}$ & $\begin{array}{c}\text { Fold } \\
\text { Change }\end{array}$ & $\begin{array}{c}P \\
\text { value }\end{array}$ & $\begin{array}{c}\text { FDR } \\
q\end{array}$ & & $\begin{array}{l}\text { Fold } \\
\text { Change }\end{array}$ & $P$ value & FDR $q$ & $\begin{array}{l}\text { Fold } \\
\text { Change }\end{array}$ & $P$ value & FDR $q$ \\
\hline $\begin{array}{l}\text { hsa-miR- } \\
\text { 106a_st }\end{array}$ & $\begin{array}{c}\text { hsa-mir- } \\
106 \mathrm{a}\end{array}$ & $\begin{array}{l}X: 133304228- \\
133304308 \\
(-)\end{array}$ & 100 & 1.5 & .036 & 0.83 & $\begin{array}{c}\text { hsa-mir- } \\
106 \mathrm{a}\end{array}$ & 1.1 & .82 & 0.90 & -1.3 & $\begin{array}{l}8.24 \times \\
10^{-14}\end{array}$ & $\begin{array}{r}7.82 \times \\
10^{-13}\end{array}$ \\
\hline $\begin{array}{l}\text { hsa-miR- } \\
\text { 377-star_ } \\
\text { st }\end{array}$ & $\begin{array}{l}\text { hsa-mir- } \\
377\end{array}$ & $\begin{array}{l}14: 101528387- \\
101528455 \\
(+)\end{array}$ & 25 & 1.7 & .036 & 0.83 & $\begin{array}{c}\text { hsa-mir- } \\
377\end{array}$ & 1.1 & .030 & 0.15 & -1.0 & .022 & 0.048 \\
\hline \multirow[t]{2}{*}{$\begin{array}{l}\text { hsa-miR- } \\
\text { 1_st }\end{array}$} & $\begin{array}{c}\text { hsa-mir- } \\
1-1\end{array}$ & $\begin{array}{l}\text { 20:61151513- } \\
61151583 \\
(+)\end{array}$ & 46 & 1.9 & .042 & 0.83 & $\begin{array}{c}\text { hsa-mir- } \\
1-1\end{array}$ & 33.8 & $\begin{array}{l}1.07 \times \\
10^{-13}\end{array}$ & $\begin{array}{c}2.25 \times \\
10^{-11}\end{array}$ & 42.1 & $\begin{array}{l}6.80 \times \\
10^{-43}\end{array}$ & $\begin{array}{c}4.67 \times \\
10^{-41}\end{array}$ \\
\hline & $\begin{array}{c}\text { hsa-mir- } \\
1-2\end{array}$ & $\begin{array}{c}18: 19408965- \\
19409049 \\
(-)\end{array}$ & & & & & $\begin{array}{c}\text { hsa-mir- } \\
1-2\end{array}$ & 32.8 & $\begin{array}{l}2.69 \times \\
10^{-13}\end{array}$ & $\begin{array}{c}3.60 \times \\
10^{-11}\end{array}$ & 43.4 & $\begin{array}{r}7.43 \times \\
10^{-43}\end{array}$ & $\begin{array}{c}4.90 \times \\
10^{-41}\end{array}$ \\
\hline $\begin{array}{l}\text { hsa-miR- } \\
4298 \text { st }\end{array}$ & $\begin{array}{c}\text { hsa-mir- } \\
4298\end{array}$ & $\begin{array}{c}11: 1880694- \\
1880766 \\
(-)\end{array}$ & 100 & 1.5 & .045 & 0.83 & $\begin{array}{c}\text { hsa-mir- } \\
4298\end{array}$ & 1.0 & & & 1.0 & & \\
\hline $\begin{array}{l}\text { hsa-let- } \\
\text { 7c_st }\end{array}$ & $\begin{array}{l}\text { hsa-let- } \\
7 \mathrm{c}\end{array}$ & $\begin{array}{l}21: 17912148- \\
17912231 \\
(+)\end{array}$ & 100 & 1.4 & .046 & 0.83 & hsa-let-7c & 1.8 & .22 & 0.51 & 1.2 & .45 & 0.55 \\
\hline $\begin{array}{c}\text { hsa-miR- } \\
17 \_s t\end{array}$ & $\begin{array}{c}\text { hsa-mir- } \\
17\end{array}$ & $\begin{array}{c}\text { 13:92002859- } \\
92002942 \\
(+)\end{array}$ & 100 & 1.5 & .048 & 0.83 & hsa-mir-17 & -1.2 & .25 & 0.55 & -1.2 & .0006 & 0.0018 \\
\hline $\begin{array}{l}\text { hsa-miR- } \\
\text { 27a-star_ } \\
\text { st }\end{array}$ & $\begin{array}{l}\text { hsa-mir- } \\
27 \mathrm{a}\end{array}$ & $\begin{array}{c}19: 13947254- \\
13947331 \\
(-)\end{array}$ & 89 & 2.4 & .050 & 0.83 & $\begin{array}{c}\text { hsa-mir- } \\
27 \mathrm{a}\end{array}$ & 1.4 & .00028 & 0.0040 & 1.4 & $\begin{array}{l}2.09 \times \\
10^{-25}\end{array}$ & $\begin{array}{c}4.65 \times \\
10^{-24}\end{array}$ \\
\hline $\begin{array}{l}\text { hsa-miR- } \\
\text { 1301_st }\end{array}$ & $\begin{array}{c}\text { hsa-mir- } \\
1301\end{array}$ & $\begin{array}{l}2: 25551509- \\
25551590 \\
(-)\end{array}$ & 100 & -2.8 & .049 & 0.83 & $\begin{array}{c}\text { hsa-mir- } \\
1301\end{array}$ & -2.3 & $\begin{array}{r}2.72 \times \\
10^{-9}\end{array}$ & $\begin{array}{r}1.53 \times \\
10^{-7}\end{array}$ & -2.2 & $\begin{array}{c}1.38 \times \\
10^{-57}\end{array}$ & $\begin{array}{c}2.84 \times \\
10^{-55}\end{array}$ \\
\hline $\begin{array}{l}\text { hsa-miR- } \\
\text { 3065-3p_ } \\
\text { st }\end{array}$ & $\begin{array}{c}\text { hsa-mir- } \\
3065\end{array}$ & $\begin{array}{c}17: 79099677- \\
79099755 \\
(+)\end{array}$ & 39 & -1.9 & .048 & 0.83 & $\begin{array}{c}\text { hsa-mir- } \\
3065\end{array}$ & -1.9 & .0024 & 0.022 & -1.6 & $\begin{array}{c}4.53 \times \\
10^{-19}\end{array}$ & $\begin{array}{r}6.62 \times \\
10^{-18}\end{array}$ \\
\hline \multirow[t]{3}{*}{$\begin{array}{l}\text { hsa-miR- } \\
\text { 3180-3p_- } \\
\text { st }\end{array}$} & $\begin{array}{l}\text { hsa-mir- } \\
\text { 3180-1 }\end{array}$ & $\begin{array}{c}16: 15005077- \\
15005170 \\
(+)\end{array}$ & 89 & -1.4 & .046 & 0.83 & $\begin{array}{l}\text { hsa-mir- } \\
\text { 3180-1 }\end{array}$ & -1.0 & .60 & 0.76 & 1.0 & .97 & 0.98 \\
\hline & $\begin{array}{l}\text { hsa-mir- } \\
\text { 3180-2 }\end{array}$ & $\begin{array}{c}16: 16403736- \\
16403823 \\
(+)\end{array}$ & & & & & $\begin{array}{l}\text { hsa-mir- } \\
\text { 3180-2 }\end{array}$ & 1.0 & & & 1.0 & .84 & 0.88 \\
\hline & $\begin{array}{l}\text { hsa-mir- } \\
\quad 3180-3\end{array}$ & $\begin{array}{c}16: 18496035- \\
18496128 \\
(-)\end{array}$ & & & & & $\begin{array}{l}\text { hsa-mir- } \\
\quad 3180-3\end{array}$ & 1.0 & .055 & 0.21 & -1.0 & .79 & 0.85 \\
\hline $\begin{array}{r}\text { hsa-miR- } \\
29 \text { a_st }\end{array}$ & $\begin{array}{l}\text { hsa-mir- } \\
29 a\end{array}$ & $\begin{array}{c}7: 130561506- \\
130561569 \\
(-)\end{array}$ & 100 & -1.3 & .042 & 0.83 & $\begin{array}{c}\text { hsa-mir- } \\
29 a\end{array}$ & -1.0 & .63 & 0.78 & 1.0 & .77 & 0.84 \\
\hline \multirow[t]{3}{*}{$\begin{array}{l}\text { hsa-miR- } \\
\text { 509-3p_ } \\
\text { st }\end{array}$} & $\begin{array}{r}\text { hsa-mir- } \\
509-1\end{array}$ & $\begin{array}{l}X: 146342050- \\
146342143 \\
(-)\end{array}$ & 29 & -2.8 & .042 & 0.83 & $\begin{array}{r}\text { hsa-mir- } \\
509-1\end{array}$ & -4.3 & .010 & 0.071 & -1.2 & $\begin{array}{l}4.53 \times \\
10^{-13}\end{array}$ & $\begin{array}{c}4.04 \times \\
10^{-12}\end{array}$ \\
\hline & $\begin{array}{r}\text { hsa-mir- } \\
509-2\end{array}$ & $\begin{array}{c}X: 146340278- \\
146340368 \\
(-)\end{array}$ & & & & & $\begin{array}{r}\text { hsa-mir- } \\
509-2\end{array}$ & -4.3 & .0014 & 0.015 & -1.2 & $\begin{array}{c}1.29 \times \\
10^{-12}\end{array}$ & $\begin{array}{l}1.11 \times \\
10^{-11}\end{array}$ \\
\hline & $\begin{array}{r}\text { hsa-mir- } \\
509-3\end{array}$ & $\begin{array}{l}X: 146341170- \\
146341244 \\
(-)\end{array}$ & & & & & $\begin{array}{r}\text { hsa-mir- } \\
509-3\end{array}$ & -4.5 & .00071 & 0.0084 & -1.1 & $\begin{array}{l}2.54 \times \\
10^{-12}\end{array}$ & $\begin{array}{c}2.11 \times \\
10^{-11}\end{array}$ \\
\hline
\end{tabular}


TABLE 2. Continued

\begin{tabular}{|c|c|c|c|c|c|c|c|c|c|c|c|c|c|}
\hline \multicolumn{7}{|c|}{ Affymetrix miRNA v3.0 } & \multirow[b]{2}{*}{$\begin{array}{c}\text { miRBase } \\
\text { v21 } \\
\text { transcript } \\
\text { ID }\end{array}$} & \multicolumn{3}{|c|}{ Illumina GA } & \multicolumn{3}{|c|}{ Illumina HiSeq } \\
\hline $\begin{array}{l}\text { Probeset } \\
\text { ID }\end{array}$ & $\begin{array}{c}\text { Transcript } \\
\text { ID(s) }\end{array}$ & $\begin{array}{l}\text { Alignment(s) } \\
\text { (hg19) }\end{array}$ & $\begin{array}{c}\text { Probeset } \\
\text { Present } \\
(\% \\
\text { samples })\end{array}$ & $\begin{array}{c}\text { Fold } \\
\text { Change }\end{array}$ & $\begin{array}{c}P \\
\text { value }\end{array}$ & $\begin{array}{c}\text { FDR } \\
q\end{array}$ & & $\begin{array}{c}\text { Fold } \\
\text { Change }\end{array}$ & $P$ value & FDR $q$ & $\begin{array}{c}\text { Fold } \\
\text { Change }\end{array}$ & $P$ value & FDR $q$ \\
\hline $\begin{array}{l}\text { hsa-miR- } \\
769-5 p_{-} \\
\text {st }\end{array}$ & $\begin{array}{c}\text { hsa-mir- } \\
769\end{array}$ & $\begin{array}{c}19: 46522190- \\
46522307 \\
(+)\end{array}$ & 100 & -2.6 & .040 & 0.83 & $\begin{array}{c}\text { hsa-mir- } \\
769\end{array}$ & -1.5 & .000086 & 0.0015 & -1.6 & $\begin{array}{c}6.51 \times \\
10^{-35}\end{array}$ & $\begin{array}{r}2.50 \times \\
10^{-33}\end{array}$ \\
\hline $\begin{array}{l}\text { hsa-miR- } \\
\text { 28-3p_st }\end{array}$ & $\begin{array}{c}\text { hsa-mir- } \\
28\end{array}$ & $\begin{array}{c}3: 188406569- \\
188406654 \\
(+)\end{array}$ & 100 & -1.6 & .038 & 0.83 & $\begin{array}{c}\text { hsa-mir- } \\
28\end{array}$ & -1.3 & .00076 & 0.0089 & -1.4 & $\begin{array}{c}5.89 \times \\
10^{-30}\end{array}$ & $\begin{array}{r}1.87 \times \\
10^{-28}\end{array}$ \\
\hline \multirow[t]{4}{*}{$\begin{array}{l}\text { hsa-miR- } \\
\text { 548i_st }\end{array}$} & $\begin{array}{l}\text { hsa-mir- } \\
548 \mathrm{i}-1\end{array}$ & $\begin{array}{c}3: 125509247- \\
125509395 \\
(-)\end{array}$ & 43 & -1.2 & .038 & 0.83 & $\begin{array}{l}\text { hsa-mir- } \\
548 \mathrm{i}-1\end{array}$ & 1.0 & & & 1.0 & & \\
\hline & $\begin{array}{l}\text { hsa-mir- } \\
548 \mathrm{i}-2\end{array}$ & $\begin{array}{c}4: 9557789- \\
9557937 \\
(-)\end{array}$ & & & & & $\begin{array}{l}\text { hsa-mir- } \\
548 \mathrm{i}-2\end{array}$ & 1.0 & & & 1.0 & & \\
\hline & $\begin{array}{l}\text { hsa-mir- } \\
548 \mathrm{i}-3\end{array}$ & $\begin{array}{l}8: 7946463- \\
7946611 \\
(-)\end{array}$ & & & & & $\begin{array}{l}\text { hsa-mir- } \\
548 \mathrm{i}-3\end{array}$ & 1.0 & & & 1.0 & & \\
\hline & $\begin{array}{l}\text { hsa-mir- } \\
548 \mathrm{i}-4\end{array}$ & $\begin{array}{c}X: 83480760- \\
83480836 \\
(-)\end{array}$ & & & & & $\begin{array}{r}\text { hsa-mir- } \\
548 \mathrm{i}-4\end{array}$ & 1.0 & & & 1.0 & & \\
\hline $\begin{array}{r}\text { hsa-miR- } \\
\text { 30a_st }\end{array}$ & $\begin{array}{l}\text { hsa-mir- } \\
30 \mathrm{a}\end{array}$ & $\begin{array}{c}6: 72113254- \\
72113324 \\
(-)\end{array}$ & 100 & -2.2 & .034 & 0.83 & $\begin{array}{l}\text { hsa-mir- } \\
\text { 30a }\end{array}$ & -1.7 & $\begin{array}{r}3.41 \times \\
10^{-8}\end{array}$ & $\begin{array}{r}1.67 \times \\
10^{-6}\end{array}$ & -1.7 & $\begin{array}{l}2.12 \times \\
10^{-32}\end{array}$ & $\begin{array}{c}7.77 \times \\
10^{-31}\end{array}$ \\
\hline \multirow[t]{2}{*}{$\begin{array}{l}\text { hsa-miR- } \\
\text { 181a_st }\end{array}$} & $\begin{array}{l}\text { hsa-mir- } \\
181 \mathrm{a}-1\end{array}$ & $\begin{array}{c}1: 198828173- \\
198828282 \\
(-)\end{array}$ & 100 & -1.6 & .025 & 0.78 & $\begin{array}{l}\text { hsa-mir- } \\
181 \mathrm{a}-1\end{array}$ & -1.3 & .00009 & 0.0015 & -1.4 & $\begin{array}{l}1.30 \times \\
10^{-25}\end{array}$ & $\begin{array}{r}2.94 \times \\
10^{-24}\end{array}$ \\
\hline & $\begin{array}{l}\text { hsa-mir- } \\
181 \mathrm{a}-2\end{array}$ & $\begin{array}{l}9: 127454721- \\
127454830 \\
(+)\end{array}$ & & & & & $\begin{array}{l}\text { hsa-mir- } \\
181 \mathrm{a}-2\end{array}$ & -1.4 & .00038 & 0.0050 & -1.2 & $\begin{array}{r}1.29 \times \\
10^{-8}\end{array}$ & $\begin{array}{r}7.38 \times \\
10^{-8}\end{array}$ \\
\hline $\begin{array}{l}\text { hsa-miR- } \\
\text { 339-3p_ } \\
\text { st }\end{array}$ & $\begin{array}{c}\text { hsa-mir- } \\
339\end{array}$ & $\begin{array}{c}7: 1062569- \\
1062662 \\
(-)\end{array}$ & 93 & -1.5 & .025 & 0.78 & $\begin{array}{c}\text { hsa-mir- } \\
339\end{array}$ & 1.3 & .62 & 0.78 & -1.2 & $\begin{array}{r}1.72 \times \\
10^{-7}\end{array}$ & $\begin{array}{r}8.64 \times \\
10^{-7}\end{array}$ \\
\hline \multirow[t]{2}{*}{$\begin{array}{l}\text { hsa-miR- } \\
\text { 548aa_st }\end{array}$} & $\begin{array}{l}\text { hsa-mir- } \\
\text { 548aa-1 }\end{array}$ & $\begin{array}{r}8: 124360274- \\
124360370 \\
(+)\end{array}$ & 89 & -1.7 & .025 & 0.78 & $\begin{array}{l}\text { hsa-mir- } \\
\text { 548aa-1 }\end{array}$ & 1.0 & & & 1.0 & & \\
\hline & $\begin{array}{l}\text { hsa-mir- } \\
548 \text { aa-2 }\end{array}$ & $\begin{array}{c}17: 65467605- \\
65467701 \\
(+)\end{array}$ & & & & & $\begin{array}{l}\text { hsa-mir- } \\
\text { 548aa-2 }\end{array}$ & 1.0 & & & -1.0 & .26 & 0.37 \\
\hline $\begin{array}{l}\text { hsa-miR- } \\
1238 \_s t\end{array}$ & $\begin{array}{c}\text { hsa-mir- } \\
1238\end{array}$ & $\begin{array}{c}19: 10662798- \\
10662880 \\
(+)\end{array}$ & 100 & -2.2 & .022 & 0.78 & $\begin{array}{c}\text { hsa-mir- } \\
1238\end{array}$ & 1.0 & .064 & 0.23 & -1.0 & .0017 & 0.0047 \\
\hline $\begin{array}{l}\text { hsa-miR- } \\
\quad 146 \mathrm{~b}-3 p_{-} \\
\text {st }\end{array}$ & $\begin{array}{c}\text { hsa-mir- } \\
146 b\end{array}$ & $\begin{array}{c}\text { 10:104196269- } \\
104196341 \\
(+)\end{array}$ & 39 & -1.9 & .013 & 0.69 & $\begin{array}{c}\text { hsa-mir- } \\
146 \mathrm{~b}\end{array}$ & -2.0 & $\begin{array}{r}6.63 \times \\
10^{-9}\end{array}$ & $\begin{array}{l}3.60 \\
\quad \times 10^{-7}\end{array}$ & -1.3 & $\begin{array}{r}3.14 \times \\
10^{-9}\end{array}$ & $\begin{array}{r}1.94 \times \\
10^{-8}\end{array}$ \\
\hline \multirow[t]{2}{*}{$\begin{array}{r}\text { hsa-miR- } \\
138 \text { st }\end{array}$} & $\begin{array}{r}\text { hsa-mir- } \\
138-1\end{array}$ & $\begin{array}{l}3: 44155704- \\
44155802 \\
(+)\end{array}$ & 100 & -3.1 & .012 & 0.69 & $\begin{array}{r}\text { hsa-mir- } \\
138-1\end{array}$ & 1.0 & .39 & 0.68 & -1.3 & $\begin{array}{r}1.77 \times \\
10^{-6}\end{array}$ & $\begin{array}{r}7.92 \times \\
10^{-6}\end{array}$ \\
\hline & $\begin{array}{r}\text { hsa-mir- } \\
138-2\end{array}$ & $\begin{array}{c}16: 56892430- \\
56892513 \\
(+)\end{array}$ & & & & & $\begin{array}{r}\text { hsa-mir- } \\
138-2\end{array}$ & -1.1 & .15 & 0.41 & -1.2 & $\begin{array}{r}1.18 \times \\
10^{-7}\end{array}$ & $\begin{array}{r}5.99 \times \\
10^{-7}\end{array}$ \\
\hline $\begin{array}{l}\text { hsa-miR- } \\
\text { 500a_st }\end{array}$ & $\begin{array}{l}\text { hsa-mir- } \\
500 \mathrm{a}\end{array}$ & $\begin{array}{c}X: 49773039- \\
49773122 \\
(+)\end{array}$ & 100 & -1.5 & .011 & 0.69 & $\begin{array}{c}\text { hsa-mir- } \\
500 \mathrm{a}\end{array}$ & -1.3 & .23 & 0.52 & -1.2 & $\begin{array}{r}1.08 \times \\
10^{-7}\end{array}$ & $\begin{array}{r}5.51 \times \\
10^{-7}\end{array}$ \\
\hline
\end{tabular}


TABLE 2. Continued

\begin{tabular}{|c|c|c|c|c|c|c|c|c|c|c|c|c|c|}
\hline \multicolumn{7}{|c|}{ Affymetrix miRNA v3.0 } & \multirow[b]{2}{*}{$\begin{array}{c}\text { miRBase } \\
\text { v21 } \\
\text { transcript } \\
\text { ID }\end{array}$} & \multicolumn{3}{|c|}{ Illumina GA } & \multicolumn{3}{|c|}{ Illumina HiSeq } \\
\hline $\begin{array}{c}\text { Probeset } \\
\text { ID }\end{array}$ & $\begin{array}{l}\text { Transcript } \\
\text { ID(s) }\end{array}$ & $\begin{array}{l}\text { Alignment(s) } \\
\text { (hg19) }\end{array}$ & $\begin{array}{c}\text { Probeset } \\
\text { Present } \\
(\% \\
\text { samples })\end{array}$ & $\begin{array}{c}\text { Fold } \\
\text { Change }\end{array}$ & $\begin{array}{c}P \\
\text { value }\end{array}$ & $\begin{array}{c}\text { FDR } \\
q\end{array}$ & & $\begin{array}{c}\text { Fold } \\
\text { Change }\end{array}$ & $P$ value & FDR $q$ & $\begin{array}{c}\text { Fold } \\
\text { Change }\end{array}$ & $P$ value & FDR $q$ \\
\hline $\begin{array}{l}\text { hsa-miR- } \\
\text { 532-5p_- } \\
\text { st }\end{array}$ & $\begin{array}{l}\text { hsa-mir- } \\
532\end{array}$ & $\begin{array}{c}X: 49767754- \\
49767844 \\
(+)\end{array}$ & 100 & -1.3 & .011 & 0.69 & $\begin{array}{l}\text { hsa-mir- } \\
532\end{array}$ & -1.3 & .067 & 0.24 & -1.6 & $\begin{array}{c}2.99 \times \\
10^{-31}\end{array}$ & $\begin{array}{r}1.05 \times \\
10^{-29}\end{array}$ \\
\hline $\begin{array}{r}\text { hsa-miR- } \\
143 \_s t\end{array}$ & $\begin{array}{c}\text { hsa-mir- } \\
143\end{array}$ & $\begin{array}{l}5: 148808481- \\
148808586 \\
\quad(+)\end{array}$ & 100 & -1.5 & .010 & 0.69 & $\begin{array}{c}\text { hsa-mir- } \\
143\end{array}$ & -1.0 & .57 & 0.76 & 1.3 & .059 & 0.11 \\
\hline $\begin{array}{l}\text { hsa-miR- } \\
\text { 30d_st }\end{array}$ & $\begin{array}{l}\text { hsa-mir- } \\
\text { 30d }\end{array}$ & $\begin{array}{l}8: 135817119- \\
135817188 \\
(-)\end{array}$ & 100 & -1.8 & .0072 & 0.69 & $\begin{array}{l}\text { hsa-mir- } \\
\text { 30d }\end{array}$ & -1.4 & .0041 & 0.035 & -1.3 & $\begin{array}{l}1.02 \times \\
10^{-14}\end{array}$ & $\begin{array}{l}1.09 \times \\
10^{-13}\end{array}$ \\
\hline $\begin{array}{l}\text { hsa-miR- } \\
\text { 425-star_ } \\
\text { st }\end{array}$ & $\begin{array}{l}\text { hsa-mir- } \\
425\end{array}$ & $\begin{array}{c}3: 49057581- \\
49057667 \\
(-)\end{array}$ & 100 & -2.3 & .0053 & 0.69 & $\begin{array}{l}\text { hsa-mir- } \\
425\end{array}$ & 1.3 & .029 & 0.15 & 1.1 & .13 & 0.22 \\
\hline $\begin{array}{l}\text { hsa-miR- } \\
\text { 34a_st }\end{array}$ & $\begin{array}{c}\text { hsa-mir- } \\
34 \mathrm{a}\end{array}$ & $\begin{array}{l}\text { 1:9211727- } \\
\quad 9211836 \\
(-)\end{array}$ & 100 & -1.4 & .0039 & 0.69 & $\begin{array}{l}\text { hsa-mir- } \\
34 \mathrm{a}\end{array}$ & -1.3 & .0032 & 0.029 & -1.4 & $\begin{array}{l}2.45 \times \\
10^{-26}\end{array}$ & $\begin{array}{l}5.86 \times \\
10^{-25}\end{array}$ \\
\hline $\begin{array}{l}\text { hsa-miR- } \\
\text { 210_st }\end{array}$ & $\begin{array}{l}\text { hsa-mir- } \\
210\end{array}$ & $\begin{array}{l}\text { 11:568089- } \\
\begin{array}{l}568198 \\
(-)\end{array}\end{array}$ & 100 & -1.8 & .0024 & 0.68 & $\begin{array}{l}\text { hsa-mir- } \\
210\end{array}$ & -1.8 & $\begin{array}{r}1.74 \times \\
10^{-6}\end{array}$ & $\begin{array}{r}6.22 \times \\
10^{-5}\end{array}$ & -2.0 & $\begin{array}{r}6.00 \times \\
10^{-28}\end{array}$ & $\begin{array}{l}1.62 \times \\
10^{-26}\end{array}$ \\
\hline $\begin{array}{l}\text { hsa-miR- } \\
\quad 4793-3 p_{-} \\
\text {st }\end{array}$ & $\begin{array}{c}\text { hsa-mir- } \\
4793\end{array}$ & $\begin{array}{c}3: 48681627- \\
48681713 \\
(-)\end{array}$ & 93 & -5.0 & .00051 & 0.22 & $\begin{array}{c}\text { hsa-mir- } \\
4793\end{array}$ & -1.1 & .077 & 0.26 & -1.0 & .00034 & 0.0011 \\
\hline $\begin{array}{l}\text { hsa-miR- } \\
\text { 4440_st }\end{array}$ & $\begin{array}{c}\text { hsa-mir- } \\
4440\end{array}$ & $\begin{array}{l}2: 239990513- \\
239990610 \\
(-)\end{array}$ & 71 & -4.0 & .00031 & 0.18 & $\begin{array}{c}\text { hsa-mir- } \\
4440\end{array}$ & 1.0 & & & -1.0 & .66 & 0.74 \\
\hline $\begin{array}{r}\text { hsa-miR- } \\
10 \mathrm{a} \_s t\end{array}$ & $\begin{array}{l}\text { hsa-mir- } \\
10 \mathrm{a}\end{array}$ & $\begin{array}{c}17: 46657200- \\
46657309 \\
(-)\end{array}$ & $96 \%$ & -3.5 & .000045 & 0.039 & $\begin{array}{c}\text { hsa-mir- } \\
10 \mathrm{a}\end{array}$ & -4.8 & $\begin{array}{c}6.13 \times \\
10^{-36}\end{array}$ & $\begin{array}{r}4.50 \times \\
10^{-33}\end{array}$ & -5.3 & $\begin{array}{l}4.80 \times \\
10^{-169}\end{array}$ & $\begin{array}{l}7.92 \times \\
10^{-166}\end{array}$ \\
\hline $\begin{array}{l}\text { imary HN } \\
\text { sample } t \\
\text { th signed } \\
t \text { statistic } \\
D R \text {, false }\end{array}$ & $\begin{array}{l}\text { puted from t } \\
\text { very rate. }\end{array}$ & r lung squamous & . & 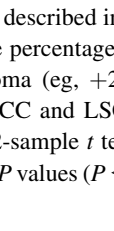 & 0 & 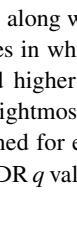 & 1 & ? & 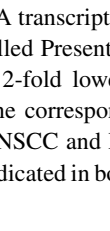 & 1. & IDs & $\operatorname{miRB}$ & $\begin{array}{l}\text { between } \\
\text { Student } \\
21 \text {, along }\end{array}$ \\
\hline
\end{tabular}

different between the 2 technologies, with the ratios measured by qPCR generally much lower than those measured by microarray.

\section{DISCUSSION}

Distinguishing between a primary LSCC and a metastatic lesion in a patient with a history of HNSCC is essential for determining cancer stage, prognosis, and therapeutic approach. However, this differentiation is difficult even after histopathologic assessment, the gold standard for the diagnosis of cancer. Patients with a history of HNSCC and the presence of lung metastases are considered to have advanced-stage cancers, and treatment will usually be aimed at palliation. ${ }^{10}$ In contrast, if the same patient is diagnosed with a primary LSCC, this would be considered an early-stage lung cancer, which will have a better prognosis, with treatment focused on surgical resection with curative intent. ${ }^{11}$ This differentiation also will help determine the appropriate surgical extent: for cases with lung metastases from HNSCC, a tissue-sparing, nonanatomical resection is indicated, such as metastasectomy or wedge resection, whereas the standard of care for a primary, early-stage lung cancer is a more extended or anatomical resection such as segmentectomy, lobectomy, or pneumonectomy, which have better survival rates but still possess considerable risk for complications. ${ }^{4}$ Despite some limitations in the published literature, metastasectomy has shown to be beneficial in selected cases, with better survival rates compared with cases managed without surgery. ${ }^{10,12-14}$ These favorable results highlight the importance of having an accurate diagnosis that will help surgeons in the selection of the appropriate surgical approach and surgical resection extent. 
TABLE 3. Performance of the miR-10a:miR-10b ratio in discriminating LSCC and HNSCC

\begin{tabular}{|c|c|c|c|c|c|c|c|c|c|}
\hline Platform & $\begin{array}{c}\text { Exclude samples with } \\
\text { prior/unknown Hx } \\
\text { of cancer or therapy? }\end{array}$ & HPV status & HNSCC & AUC & Accuracy & Sensitivity & Specificity & PPV & NPV \\
\hline \multirow{2}{*}{$\begin{array}{l}\text { Affymetrix } \\
\text { miRNA v3.0 }\end{array}$} & \multirow[t]{2}{*}{ Yes } & All & All & 0.922 & $78.6 \%$ & $93.8 \%$ & $58.3 \%$ & $75.0 \%$ & $87.5 \%$ \\
\hline & & Negative & All & 0.969 & $85.0 \%$ & $93.8 \%$ & $50.0 \%$ & $88.2 \%$ & $66.7 \%$ \\
\hline \multirow{8}{*}{$\begin{array}{l}\text { Illumina Genome } \\
\text { Analyzer }\end{array}$} & \multirow[t]{4}{*}{ No } & \multirow[t]{2}{*}{ All } & All & 0.970 & $81.5 \%$ & $77.2 \%$ & $97.3 \%$ & $99.1 \%$ & $53.7 \%$ \\
\hline & & & Stage IV only & 0.971 & $80.9 \%$ & $77.2 \%$ & $100.0 \%$ & $100.0 \%$ & $45.6 \%$ \\
\hline & & \multirow[t]{2}{*}{ Negative } & All & 0.964 & $80.4 \%$ & $77.2 \%$ & $96.3 \%$ & $99.1 \%$ & $45.6 \%$ \\
\hline & & & Stage IV only & 0.967 & $80.0 \%$ & $77.2 \%$ & $100.0 \%$ & $100.0 \%$ & $38.0 \%$ \\
\hline & \multirow[t]{4}{*}{ Yes } & \multirow[t]{2}{*}{ All } & All & 0.975 & $83.7 \%$ & $79.5 \%$ & $97.1 \%$ & $98.9 \%$ & $59.6 \%$ \\
\hline & & & Stage IV only & 0.978 & $83.1 \%$ & $79.5 \%$ & $100.0 \%$ & $100.0 \%$ & $51.1 \%$ \\
\hline & & \multirow[t]{2}{*}{ Negative } & All & 0.969 & $82.5 \%$ & $79.5 \%$ & $96.0 \%$ & $98.9 \%$ & $51.1 \%$ \\
\hline & & & Stage IV only & 0.974 & $82.2 \%$ & $79.5 \%$ & $100.0 \%$ & $100.0 \%$ & $42.5 \%$ \\
\hline \multirow[t]{8}{*}{ Illumina HiSeq } & \multirow[t]{4}{*}{ No } & \multirow[t]{2}{*}{ All } & All & 0.982 & $93.0 \%$ & $86.2 \%$ & $97.7 \%$ & $96.4 \%$ & $91.0 \%$ \\
\hline & & & Stage IV only & 0.980 & $91.1 \%$ & $86.2 \%$ & $98.0 \%$ & $98.3 \%$ & $83.6 \%$ \\
\hline & & \multirow[t]{2}{*}{ Negative } & All & 0.982 & $92.5 \%$ & $86.2 \%$ & $98.0 \%$ & $97.3 \%$ & $89.2 \%$ \\
\hline & & & Stage IV only & 0.980 & $90.7 \%$ & $86.2 \%$ & $98.1 \%$ & $98.7 \%$ & $81.3 \%$ \\
\hline & \multirow[t]{4}{*}{ Yes } & \multirow[t]{2}{*}{ All } & All & 0.980 & $92.8 \%$ & $84.9 \%$ & $98.0 \%$ & $96.5 \%$ & $90.9 \%$ \\
\hline & & & Stage IV only & 0.977 & $90.6 \%$ & $84.9 \%$ & $97.8 \%$ & $98.0 \%$ & $83.8 \%$ \\
\hline & & \multirow[t]{2}{*}{ Negative } & All & 0.980 & $92.3 \%$ & $84.9 \%$ & $98.1 \%$ & $97.2 \%$ & $89.2 \%$ \\
\hline & & & Stage IV only & 0.977 & $90.2 \%$ & $84.9 \%$ & $98.0 \%$ & $98.4 \%$ & $81.5 \%$ \\
\hline
\end{tabular}

The performance characteristics (AUC, accuracy, sensitivity, specificity, PPV, and NPV) are summarized according to platform, exclusion criteria, HPV status, and exclusion or inclusion of non-stage-IV HNSCC. HPV, Human papillomavirus; HNSCC, head and neck squamous cell carcinoma; AUC, area under the (receiver operating characteristics) curve; $P P V$, positive predictive value; $N P V$, negative predictive value.

At least some of the differential expression observed between tumor types appears to be attributable to contamination of the tumor samples with surrounding tissue. The set of miRNAs that are expressed more highly in HNSCC than in LSCC in our cohort includes 4 "myo-miRs" (miR-206, miR-133a, miR-133b, and miR-1) whose expression has been well-established as restricted to striated muscle, ${ }^{15}$ as well as miR-486-3p (which also has been reported to be enriched in muscle ${ }^{16}$ and is intronic to the ANKl gene, an isoform of which is specific to striated muscle and interacts with the muscle proteins $\operatorname{titin}^{17}$ and obscurin ${ }^{18}$ and miR-31, a "dystromir" that is a marker of skeletal muscle regeneration after injury ${ }^{19}$ ). In addition, 7 miRNAs that are expressed more highly in HNSCC (miR-379, miR-411, miR-299, miR-381, miR-134, miR-377*, and miR-409) are located within the DLK1-DIO3 cluster in chromosome 14, which recently has been shown to be regulated by the transcription factor myocyte enhancer factor $2,{ }^{20}$ which is essential for myocyte differentiation and skeletal muscle growth. ${ }^{21}$ The expression of all of these miRNAs also was significantly greater in HNSCC in both TCGA datasets, with the exception of 2 DLK-DIO3 cluster members (miR-379 and miR-409). Taken together, the increased expression of these miRNAs in HNSCC samples therefore likely represents contamination with surrounding striated muscle, which is unlikely to be present in LSCC specimens. Conversely, the expression of miR-34b*,
miR-34c-5p, and miR-449a/b/c, whose expression is largely restricted to multiciliated cells (MCCs) such as those in the bronchial epithelium, ${ }^{22}$ was more than 2-fold greater in LSCC than in HNSCC in our cohort and significantly (FDR $q<0.25$ ) greater in LSCC in both TCGA datasets. Importantly, however, although miR-34a shares a seed sequence with these MCC-specific miRNAs, its pattern of expression is not restricted to MCCs and is therefore unlikely to represent contamination from the airway epithelium.

Several miRNAs that were expressed more highly in HNSCC in our cohort appear to be unrelated to striated muscle contamination, however, including miR-203 $(P=.028)$, which typically is regarded as a keratinocyte-specific miRNA ${ }^{23}$ but has been detected in several squamous epithelia, including the esophagus, and is well-expressed in normal human bronchial epithelial cells grown in culture. ${ }^{24}$ Another such miRNA is $\mathrm{miR}-455-3 \mathrm{p}(P=.017)$, whose expression has been reported recently to be associated with increased survival in both HNSCC ${ }^{25}$ and NSCLC. ${ }^{26}$ The expression of both miRNAs also was robustly greater in HNSCC in both TCGA datasets (FDR $q<0.005$ ).

Previous studies have shown that profiling of the expression of messenger RNA (mRNA) may be useful to discriminate between primary and metastatic lung squamous cell carcinomas in patients with a history of HNSCC. Talbot and colleagues ${ }^{27}$ showed that an expression 


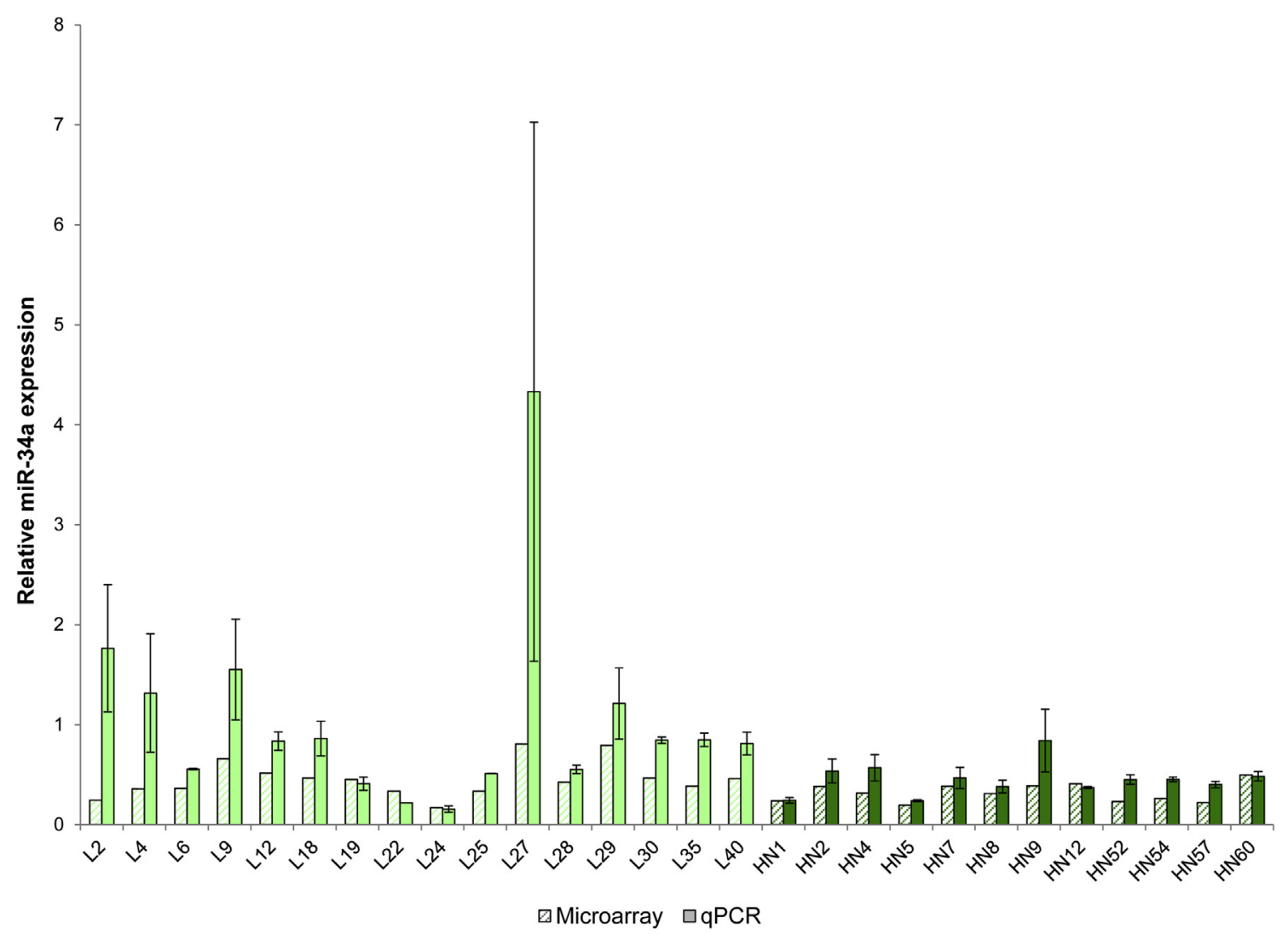

FIGURE 2. Relative expression of miR-34a. Expression of miR-34a was measured in each lung squamous cell carcinoma (light green) or head and neck squamous cell carcinoma (dark green) sample by microarray (hatched bars) and quantitative real-time polymerase chain reaction (solid bars). Microarray expression was exponentiated to convert to linear scale, and then relative expression was computed as the ratio of miR-34a to the geometric mean of miR-191-5p and miR-361-5p. Similarly, qPCR relative expression was computed relative to an interplate reference sample and then separately to miR-191-5p and miR-361-5p to obtain 2 measurements for each replicate, which were averaged together using the geometric mean. Bars for qPCR measurements indicate mean \pm SEM. $q P C R$, Quantitative real-time polymerase chain reaction.

profile of 100-500 genes could accomplish this goal. Similarly, Vachani and colleagues ${ }^{28}$ produced a 10 -gene classifier that distinguished between primary lung and metastatic lesions, but the results of that study appear to be confounded with differences in cell type composition, as the classifier includes the lung surfactant SFTPB (whose expression is greater in LSCC) and the striated muscle markers TMP3 and MYH2 (whose expression is greater in HNSCC), and the larger set of discriminators included the lung surfactant genes ( $S F T P C, S F T P D)$, airway secretory epithelial markers (SCGB1A1 and MUC5B), and an alveolar marker (ABCA3), as well as the striated muscle markers ACTA1, ANK3, MYL1, and TPM2. The authors discuss the possibility that the increased expression of the lung surfactant genes in LSCC may be attributable to contamination with nontumor lung tissue, and indicated that SFTPC in particular may be expressed by LSCC; however, they did not address the presence of the muscle-specific markers in their predictive gene sets.
The use of miRNA profiling to establish discriminators between these 2 tumor types could be a more useful and accurate technique than mRNA expression profiling, as miRNAs are more resistant to degradation and remain largely intact in FFPE samples for longer time than mRNA, and are also recognized for their tissue specificity. This possibility was explored by Rosenfeld and colleagues, ${ }^{29}$ who used miRNA expression in tumor samples to derive a decision tree to infer the primary tissue of origin. Importantly, miR-34a was 1 of only 3 miRNAs (along with miR-148b and miR-182) that were used to discriminate between LSCC and HNSCC in this tree (all 3 expressed more highly in LSCC). Although the latter 2 miRNAs were not significantly differentially expressed in our cohort, their expression was significantly greater in LSCC in both TCGA data sets (FDR $q<10^{-7}$ ). This discrepancy may be explained by the difference in sample size, but as miR-148b and miR-182 also are expressed at relatively low and high levels, respectively, the improved 


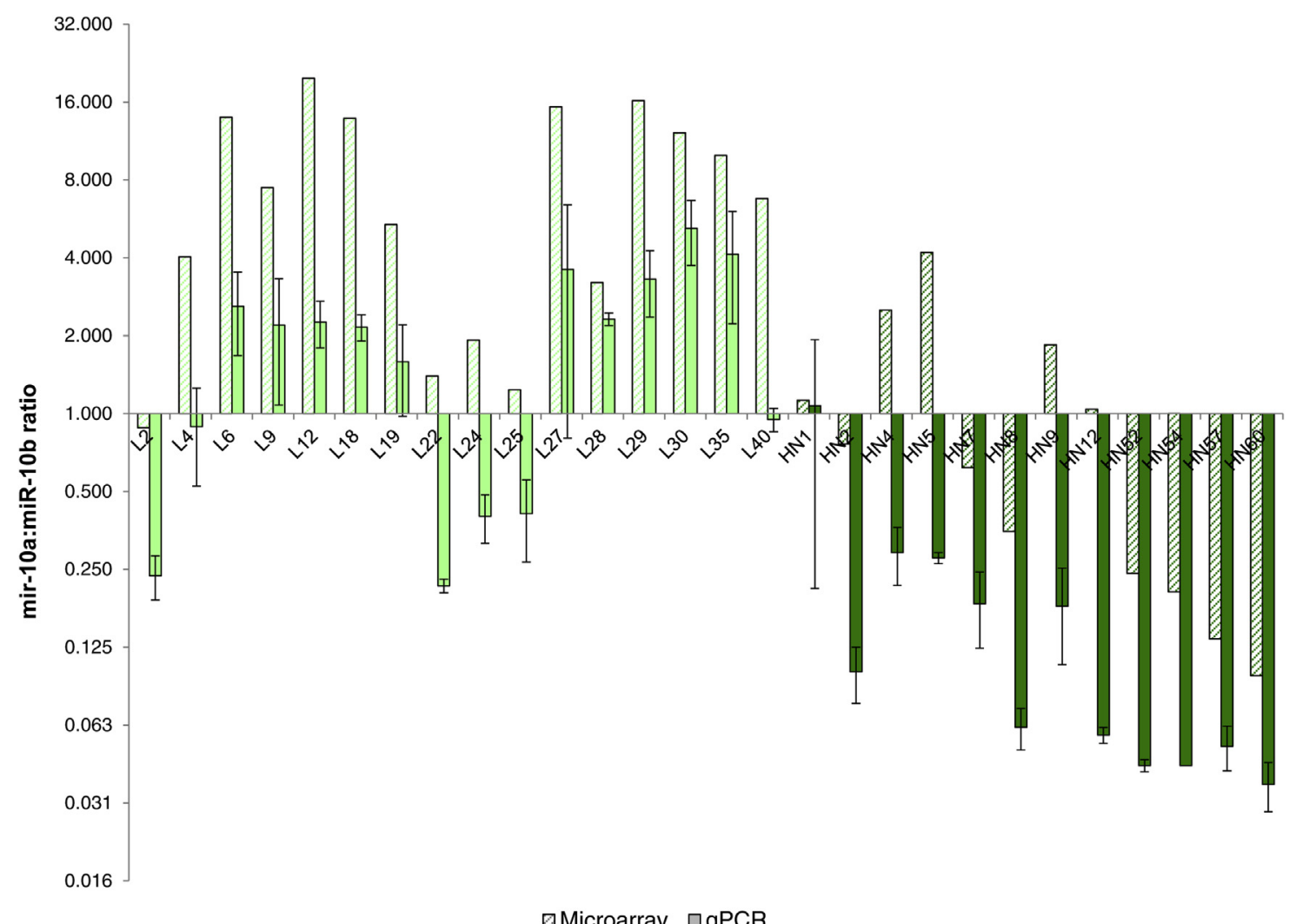

FIGURE 3. Ratio of miR-10:miR-10b. The ratio of the expression of miR-10a and miR-10b was measured in each lung squamous cell carcinoma (light green) or head and neck squamous cell carcinoma (dark green) sample by microarray (hatched bars) and quantitative real-time polymerase chain reaction (solid bars). Bars for qPCR measurements indicate mean \pm SEM. $q P C R$, Quantitative real-time polymerase chain reaction.

dynamic range and specificity of RNA sequencing also may contribute to this difference.

One of the most striking results of this study is the strong and robust association of the miR-10a:miR-10b ratio with tumor type, with the expression of the former and latter strongly increased in LSCC or HNSCC, respectively. This reciprocal relationship is not entirely surprising, as miR-10b is located $\sim 1 \mathrm{~kb}$ upstream of HOXD4, a known target of miR-10a. ${ }^{30}$ The particular advantage of using a ratio of 2 miRNAs as a discriminator is that it removes the need to select and profile an appropriate a housekeeping control, as each miRNA serves as the internal control for the other. This approach also was explored by Vachani and colleagues, ${ }^{28}$ but as all of their ratios included a lung surfactant gene (SFTPA2 or SFTPB), it is likely that their results reflect relative presence of contaminating normal lung tissue rather than biological differences between the 2 tumor types.

Although this study has demonstrated that the expression of a small number of miRNAs may be useful in clinical discrimination of primary LSCC and HNSCC tumors, several challenges remain. Contamination of tumor samples with surrounding tissue such as striated muscle or airway epithelium is a limitation that could be addressed in future studies with the use of laser capture microdissection to isolate tumor cells before profiling and increase statistical power to discriminate between tumor types, although this technique is too cumbersome to be useful in routine diagnostic practice. A more important future goal, however, is to obtain more samples, especially those from solitary lung lesions found in patients with a history of HNSCC (and, where possible, matched HNSCC samples from the same subject).

\section{CONCLUSIONS}

The distinction between a primary LSCC and a metastatic lesion in a patient with a history of HNSCC is difficult even after histopathologic assessment. The differentiation between these 2 is essential because this will determine the prognosis and the selection of the appropriate therapeutic approach. The expression of several miRNAs may be useful for discriminating between HNSCC and LSCC, including the expression of miR-34a and the ratio of the expression of miR-10a and miR-10b.

\section{Conflict of Interest Statement}

Authors have nothing to disclose with regard to commercial support. 
We thank Yuriy Alekseyev and the Boston University Microarray and Sequencing Resource for generating the raw microarray data.

\section{References}

1. Schoenwolf GC, Bleyl SB, Brauer PR, Francis-West PH. Development of the respiratory system and body cavities, development of the pharyngeal apparatus and face. In: Schoenwolf GC, Bleyl SB, Brauer PR, Francis-West PH, eds. Larsen's Human Embryology. 4th ed. Philadelphia: Churchill Livingstone/ Elsevier; 2009:319-23, 573-9.

2. Ezzati M, Henley SJ, Lopez AD, Thun MJ. Role of smoking in global and regional cancer epidemiology: current patterns and data needs. Int $J$ Cancer. 2005;116:963-71.

3. Takes RP, Rinaldo A, Silver CE, Haigentz M Jr, Woolgar JA, Triantafyllou A, et al. Distant metastases from head and neck squamous cell carcinoma. Part I. Basic aspects. Oral Oncol. 2012;48:775-9.

4. Rotman JA, Plodkowski AJ, Hayes SA, de Groot PM, Shepard JA, Munden RF, et al. Postoperative complications after thoracic surgery for lung cancer. Clin Imaging. 2015;39:735-49.

5. Almeida MI, Reis RM, Calin GA. MicroRNA history: discovery, recent applications, and next frontiers. Mutat Res. 2011;717:1-8.

6. Calin GA, Sevignani C, Dumitru CD, Hyslop T, Noch E, Yendamuri S, et al. Human microRNA genes are frequently located at fragile sites and genomic regions involved in cancers. Proc Natl Acad Sci U S A. 2004;101:2999-3004.

7. Lu J, Getz G, Miska EA, Alvarez-Saavedra E, Lamb J, Peck D, et al. MicroRNA expression profiles classify human cancers. Nature. 2005;435:834-8.

8. Calin GA, Croce CM. MicroRNA signatures in human cancers. Nat Rev Cancer. 2006;6:857-66.

9. Hellemans J, Mortier G, De Paepe A, Speleman F, Vandesompele J. qBase relative quantification framework and software for management and automated analysis of real-time quantitative PCR data. Genome Biol. 2007;8:R19.

10. Haigentz M Jr, Hartl DM, Silver CE, Langendijk JA, Strojan P, Paleri V, et al. Distant metastases from head and neck squamous cell carcinoma. Part III. Treatment. Oral Oncol. 2012;48:787-93.

11. Pearson FG. Non-small cell lung cancer: role of surgery for stages I-III. Chest. 1999;116:500S-3S.

12. Shiono S, Kawamura M, Sato T, Okumura S, Nakajima J, Yoshino I, et al. Metastatic Lung Tumor Study Group of Japan. Pulmonary metastasectomy for pulmonary metastases of head and neck squamous cell carcinomas. Ann Thorac Surg. 2009;88:856-60.

13. Hosokawa S, Funai K, Sugiyama K, Takahashi G, Okamura J, Takizawa Y, et al. Survival outcomes after surgical resection of pulmonary metastases of head and neck tumours. J Laryngol Otol. 2016;130:291-5.

14. Winter H, Meimarakis G, Hoffmann G, Hummel M, Rüttinger D, Zilbauer A, et al. Does surgical resection of pulmonary metastases of head and neck cancer improve survival? Ann Surg Oncol. 2008;15:2915-26.

15. Horak M, Novak J, Bienertova-Vasku J. Muscle-specific microRNAs in skeletal muscle development. Dev Biol. 2016;410:1-13.

16. Small EM, O'Rourke JR, Moresi V, Sutherland LB, McAnally J, Gerard RD, et al. Regulation of PI3-kinase/Akt signaling by muscle-enriched microRNA-486. Proc Natl Acad Sci USA. 2010;107:4218-23.

17. Kontrogianni-Konstantopoulos A, Bloch RJ. The hydrophilic domain of small ankyrin-1 interacts with the two N-terminal immunoglobulin domains of titin. J Biol Chem. 2003:278:3985-91.

18. Kontrogianni-Konstantopoulos A, Jones EM, Van Rossum DB, Bloch RJ. Obscurin is a ligand for small ankyrin 1 in skeletal muscle. Mol Biol Cell. 2003; $14: 1138-48$.

19. Greco S, De Simone M, Colussi C, Zaccagnini G, Fasanaro P, Pescatori M, et al. Common micro-RNA signature in skeletal muscle damage and regeneration induced by Duchenne muscular dystrophy and acute ischemia. FASEB J. 2009; 23:3335-46.

20. Snyder CM, Rice AL, Estrella NL, Held A, Kandarian SC, Naya FJ. MEF2A regulates the Gt12-Dio3 microRNA mega-cluster to modulate WNT signaling in skeletal muscle regeneration. Development. 2013;140:31-42.

21. Naya FJ, Olson E. MEF2: a transcriptional target for signaling pathways controlling skeletal muscle growth and differentiation. Curr Opin Cell Biol. 1999:11:683-8.

22. Wu J, Bao J, Kim M, Yuan S, Tang C, Zheng H, et al. Two miRNA clusters, miR-34b/ $\mathrm{c}$ and miR-449, are essential for normal brain development, motile ciliogenesis, and spermatogenesis. Proc Natl Acad Sci USA. 2014;111:E2851-7.
23. Yi R, Poy MN, Stoffel M, Fuchs E. A skin microRNA promotes differentiation by repressing 'stemness'. Nature. 2008;452:225-9.

24. Jin J, Deng J, Wang F, Xia X, Qiu T, Lu W, et al. The expression and function of microRNA-203 in lung cancer. Tumour Biol. 2013;34:349-57.

25. Wong N, Khwaja SS, Baker CM, Gay HA, Thorstad WL, Daly MD, et al. Prognostic microRNA signatures derived from The Cancer Genome Atlas for head and neck squamous cell carcinomas. Cancer Med. 2016;5:1619-28.

26. Li YJ, Ping C, Tang J, Zhang W. MicroRNA-455 suppresses non-small cell lung cancer through targeting ZEB1. Cell Biol Int. 2016;40:621-8.

27. Talbot SG, Estilo C, Maghami E, Sarkaria IS, Pham DK, O-charoenrat P, et al Gene expression profiling allows distinction between primary and metastatic squamous cell carcinomas in the lung. Cancer Res. 2005;65:3063-71.

28. Vachani A, Nebozhyn M, Singhal S, Alila L, Wakeam E, Muschel R, et al. A 10-gene classifier for distinguishing head and neck squamous cell carcinoma and lung squamous cell carcinoma. Clin Cancer Res. 2007;13:2905-15.

29. Rosenfeld N, Aharonov R, Meiri E, Rosenwald S, Spector Y, Zepeniuk M, et al. MicroRNAs accurately identify cancer tissue origin. Nat Biotechnol. 2008;26: 462-9.

30. Tan Y, Zhang B, Wu T, Skogerbø G, Zhu X, Guo X, et al. Transcriptional inhibition of Hoxd4 expression by miRNA-10a in human breast cancer cells. BMC Mol Biol. 2009; 10:12.

Key Words: microRNA, head and neck tumors, squamous cell cancer, lung metastasis, diagnosis, surgical resection

\section{Discussion}

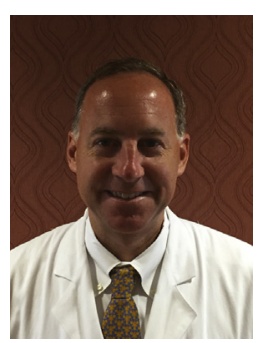

Dr Mark Onaitis (Durham, NC). Nice job. Thanks for sending me the manuscript in advance. Clearly, the Boston group is still working on leading the world in miRNA and showing how it predicts prognosis and, in this case, site of origin.

I have 3 questions. What are the targets of miR-34 and miR-10a, and does that tell us anything about the biology of lung cancer or head and neck cancer?

Dr Juan A. Munoz (Boston, Mass). Although many miRNAs have unknown targets, the targets for miR-10a and the miR-34 family have been well described. We know that the miR-10a family targets the Hox genes, which are involved in multiple developmental and embryological processes. Regarding miR-34a, we know that it targets p53 and, conversely, p53 regulates miR-34a. So this feedback could be interesting, and, yes, it is associated with these oncologic processes.

Dr Onaitis. Do you think these miRNAs represent the tissue of origin or do you think that they are part of the oncologic process, or both?

Dr Munoz. I would say both. The expression of these miRNAs might be present in the normal tissues from the head and neck or from the lung, but what is interesting here is to see the expression or the differential expression during the oncologic processes.

Dr Onaitis. Hard to know?

Dr Munoz. Yes.

Dr Onaitis. Your miRNA array was on primary head and neck tumors, but we are trying to figure out when someone 
comes in with a lung nodule and they previously had head and neck cancer, is this metastasis or is this primary? Do miRNAs change during metastasis? If you looked at actual lung lesions from head and neck metastases, would you see the same results?

Dr Munoz. We actually tried to look at the samples from the primary and from the metastatic lesions. We are looking for these patients. We don't know at this moment if the expression is the same, but we know that miR-10b is related with invasiveness in breast cancer, as reported in the literature. So we could say that maybe the expression of miR-10b is greater in the metastatic lesion. But, yes, that would be very interesting to see, and we are trying to actually find these cases with the primary lesion and the metastatic lesion to compare the miRNA signature.

Dr Onaitis. Nice job.

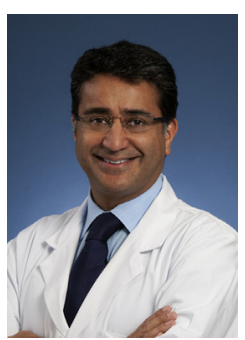

Dr Shaf Keshavjee (Toronto, Ontario, Canada). I have another question that is more philosophical: if you look at a single biomarker, you have to examine expression in the 2 tumors and then find a cutoff level. You know that most of our biomarkers to date, depending on how good they are, may be expressed to a certain degree in certain parts of tumors, but not $100 \%$. If you really wanted to specifically answer the question of: Is this tumor in the lung a metastasis from the head and neck or a new primary, wouldn't we be better looking at overall array patterns of expression to really answer that question and then go to personalizing your therapy based on what the current tumor is that you are treating? Array patterns that are so dramatically different would tell you that it is a different tumor rather than expression level comparison of one marker.

Dr Munoz. I'm not sure if I completely understand the idea. The interesting thing here is that we can see what the expression in these 2 different tumors is, but I am not sure.

Dr Keshavjee. What my question related to is, when you showed the microarrays, the first question that came into my head is what does the array of the same tumor from the head and neck and from the lung in that patient look like and how many genes were co-upregulated or down-regulated in the same tumor side by side? That would be the comparison that would be very interesting to see.

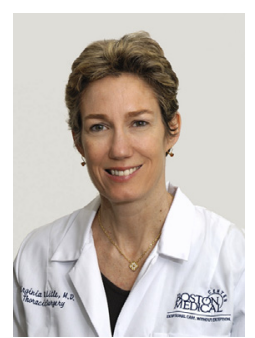

Dr Virginia Litle (Boston, Mass). I agree. When you are talking about using a biomarker to guide clinical decision-making, you really do have to have as high an accuracy rate as possible, and ours is pretty high, but I think we are also trying to work toward developing something that would be practical, and the idea is that you could do a CT-guided or a navigational bronchoscopic biopsy of the lesion, and then you could do PCR. It may end up being a panel of biomarkers, but if we can narrow it down to a few in number, we could actually use it clinically versus having a lower throughput test, like arrays.

Dr Keshavjee. Great. Good point. 